\title{
Au-Catalyzed Reaction of Propargylic Sulfides and Dithioacetals
}

\author{
Lingling Peng, Xiu Zhang, Shiwei Zhang and Jianbo Wang*
}

Beijing National Laboratory of Molecular Sciences (BNLMS), Green Chemistry Center (GCC) and Key Laboratory of Bioorganic Chemistry and Molecular Engineering of Ministry of Education, College of Chemistry, Peking University, Beijing 100871, China

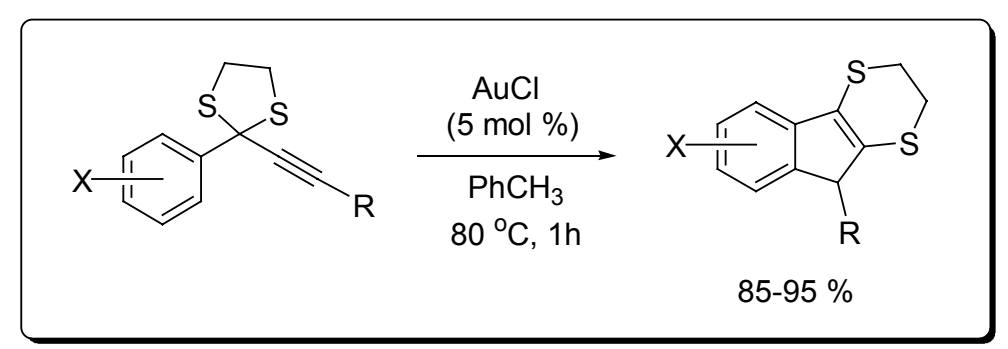

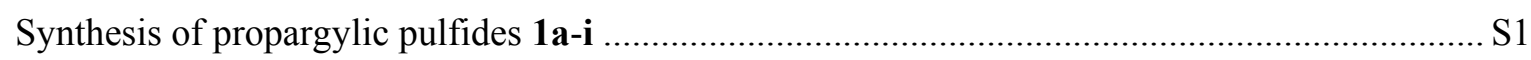

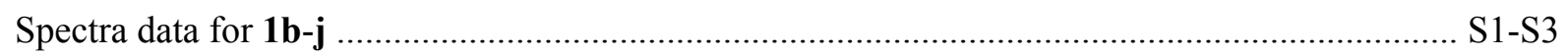

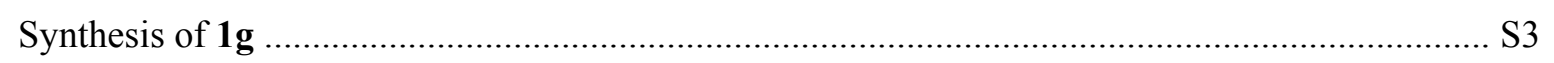

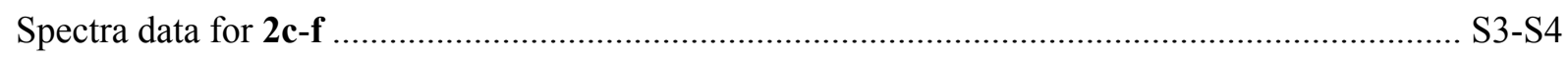

$\mathrm{X}$-ray structure of 18

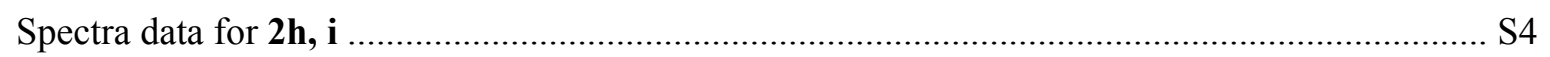

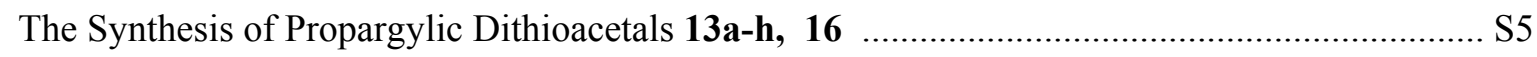

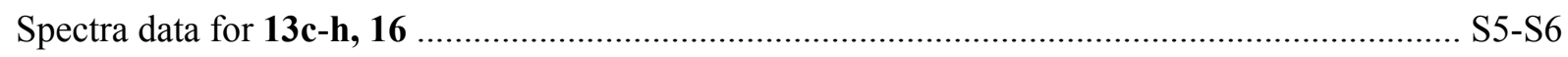

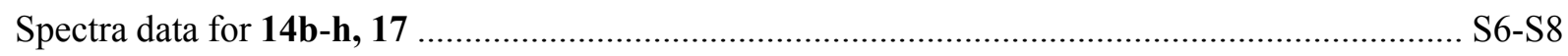

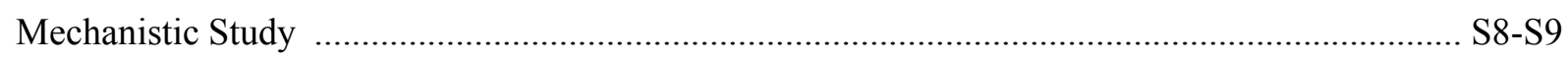

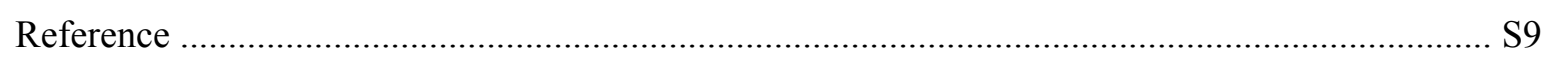

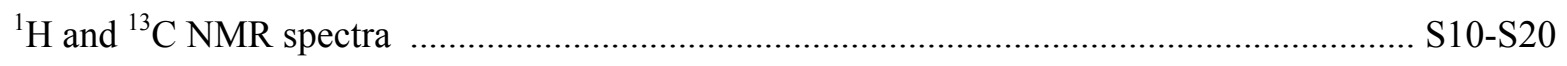




\section{Experimental Section}

General All reactions were performed under a nitrogen atmosphere in a flame-dried reaction flask. All solvents were distilled prior to use. $\mathrm{CH}_{2} \mathrm{Cl}_{2}$ was distilled over calcium hydride, and toluene was distilled over sodium.

\section{Synthesis of Propargylic Sulfides (1a-i)}

The propargylic sulfides were synthesized through catalyzed propargylic substitution reaction of propargylic alcohols with thiols. We mainly used two methods: catalysis with $\mathrm{ZnI}_{2}$ (Method A) or catalysis with PTS ( $p$-toluenesulfonic acid monohydrate) (Method B). The sulfides can also be synthesized by catalysis with thiolate-bridged diruthenium complex or $\mathrm{NaAuCl}_{4} \cdot 2 \mathrm{H}_{2} \mathrm{O}$. Recently, some other methods for preparing propargylic sulfides have appeared in literature.
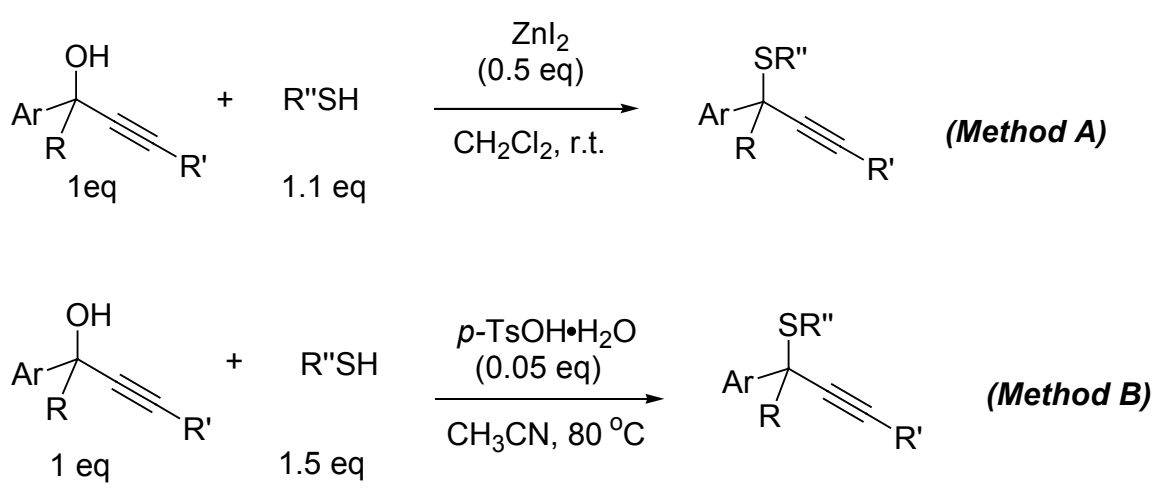

2-Chlorophenyl 1-methyl-1-phenyl-2-propynyl sulfide 1b. Yield 28 \% (Method A); IR (film) 3296, 1449, 1034, 752, $696 \mathrm{~cm}^{-1} ;{ }^{1} \mathrm{H}$ NMR $\left(\mathrm{CDCl}_{3}, 300 \mathrm{MHz}\right) \delta 1.97(\mathrm{~s}, 3 \mathrm{H}), 2.72(\mathrm{~s}, 1 \mathrm{H}), 7.06-7.13(\mathrm{~m}, 1 \mathrm{H})$, 7.15-7.21 (m, 1H), 7.21-7.39 (m, 4H), 7.44-7.48 (m, 1H), 7.65-7.70 (m, 2H); ${ }^{13} \mathrm{C}$ NMR $\left(\mathrm{CDCl}_{3}, 75\right.$ MHz) $\delta 30.6,49.8,74.8,85.6,126.5,126.6,127.7,128.2,129.5,129.6,132.1,136.5,138.5,141.4$; EIMS (m/z, relative intensity): $272\left(\mathrm{M}^{+}, 5\right), 257$ (13), 129 (100). Anal. calcd. for $\mathrm{C}_{16} \mathrm{H}_{13} \mathrm{ClS}: \mathrm{C}, 70.45 ; \mathrm{H}$, 4.80. Found: C, 70.60; H, 4.94.

2-Naphthyl 1-methyl-1-phenyl-2-propynyl sulfide 1c. Yield $20 \%$ (Method A); mp: $66-67{ }^{\circ} \mathrm{C}$; IR (film) 3292, 1496, 1067, 859, 816, 742, $696 \mathrm{~cm}^{-1}$; ${ }^{1} \mathrm{H}$ NMR $\left(\mathrm{CDCl}_{3}, 300 \mathrm{MHz}\right) \delta 1.98$ (s, 3H), 2.70 (s, 1H), 7.22-7.32 (m, 3H), 7.35-7.39 (m, 1H), 7.42-7.51 (m, 2H), 7.58-7.68 (m, 3H), 7.69-7.74 (m, 1H), 7.77-7.80 (m, 1H), $7.86(\mathrm{~s}, 1 \mathrm{H}) ;{ }^{13} \mathrm{C} \mathrm{NMR}\left(\mathrm{CDCl}_{3}, 75 \mathrm{MHz}\right) \delta 29.9,49.5,74.8,86.1,126.1,126.7$, $126.8,127.5,127.5,127.6,128.0,128.1,129.7,132.8,133.2,133.2,136.5,141.9$; EI-MS ( $\mathrm{m} / \mathrm{z}$, relative intensity): $288\left(\mathrm{M}^{+}, 53\right), 273$ (84), 160 (34), 129 (100). Anal. calcd. for $\mathrm{C}_{20} \mathrm{H}_{16} \mathrm{~S}$ : C, 83.29; H, 5.59. Found: C, 83.17; H, 5.64. 
Benzyl 1-methyl-1-phenyl-2-propynyl sulfide 1d. Yield 27 \% (Method A); IR (film) 3288, 1494, 1069, 803, 695, $684 \mathrm{~cm}^{-1} ;{ }^{1} \mathrm{H}$ NMR $\left(\mathrm{CDCl}_{3}, 300 \mathrm{MHz}\right) \delta 1.89(\mathrm{~s}, 3 \mathrm{H}), 2.78(\mathrm{~s}, 1 \mathrm{H}), 3.53(\mathrm{~d}, J=12 \mathrm{~Hz}$, $1 \mathrm{H}), 3.89(\mathrm{~d}, J=12 \mathrm{~Hz}, 1 \mathrm{H}), 7.15-7.25(\mathrm{~m}, 5 \mathrm{H}), 7.26-7.30(\mathrm{~m}, 1 \mathrm{H}), 7.32-7.38(\mathrm{~m}, 2 \mathrm{H}), 7.74-7.78(\mathrm{~m}$, $2 \mathrm{H}) ;{ }^{13} \mathrm{C} \mathrm{NMR}\left(\mathrm{CDCl}_{3}, 75 \mathrm{MHz}\right) \delta 31.1,36.2,46.6,73.8,85.8,126.4,126.9,127.4,128.3,128.3$, 129.2, 136.9, 142.2; EI-MS ( $m / z$, relative intensity): $252\left(\mathrm{M}^{+}, 8\right), 237$ (10), 161 (8), 129 (100). Anal. calcd. for $\mathrm{C}_{17} \mathrm{H}_{16} \mathrm{~S}$ : C, 80.90; H, 6.39. Found: C, 81.12; H, 6.41.

Hexyl 1-methyl-1-phenyl-2-propynyl sulfide 1e. Yield $35 \%$ (Method A); IR (film) 3306, 2927, 1599, $697 \mathrm{~cm}^{-1} ;{ }^{1} \mathrm{H}$ NMR $\left(\mathrm{CDCl}_{3}, 300 \mathrm{MHz}\right) \delta 0.84(\mathrm{t}, J=6.6 \mathrm{~Hz}, 3 \mathrm{H}), 1.15-1.33(\mathrm{~m}, 6 \mathrm{H}), 1.40-1.50$ $(\mathrm{m}, 2 \mathrm{H}), 1.88(\mathrm{~s}, 3 \mathrm{H}), 2.32-2.41(\mathrm{~m}, 1 \mathrm{H}), 2.60-2.68(\mathrm{~m}, 1 \mathrm{H}), 2.71(\mathrm{~s}, 1 \mathrm{H}), 7.22-7.27(\mathrm{~m}, 1 \mathrm{H}), 7.30-$ $7.36(\mathrm{~m}, 2 \mathrm{H}), 7.70-7.75(\mathrm{~m}, 2 \mathrm{H}) ;{ }^{13} \mathrm{C} \mathrm{NMR}\left(\mathrm{CDCl}_{3}, 75 \mathrm{MHz}\right) \delta 13.9,22.4,28.4,28.6,31.3,45.6,73.3$, 86.2, 126.4, 127.2, 128.1, 142.7; EI-MS (m/z, relative intensity): 246 (M+, 4), 161 (12), 129 (100). Anal. calcd. for $\mathrm{C}_{16} \mathrm{H}_{22} \mathrm{~S}$ : C, 77.99; H, 9.00. Found: C, 77.83; H, 9.02.

2-Chlorobenzyl 1-methyl-1-phenyl-2-propynyl sulfide 1f. Yield $15 \%$ (Method A); IR (film) 3296, 1473, 1444, 1053, 742, $697 \mathrm{~cm}^{-1} ;{ }^{1} \mathrm{H} \mathrm{NMR}\left(\mathrm{CDCl}_{3}, 300 \mathrm{MHz}\right) \delta 1.91(\mathrm{~s}, 3 \mathrm{H}), 2.80$ (s, 1H), 3.67 (d, $J=$ $12 \mathrm{~Hz}, 1 \mathrm{H}), 4.01(\mathrm{~d}, J=12 \mathrm{~Hz}, 1 \mathrm{H}), 7.05-7.17(\mathrm{~m}, 3 \mathrm{H}), 7.23-7.37(\mathrm{~m}, 4 \mathrm{H}), 7.74-7.78(\mathrm{~m}, 2 \mathrm{H}) ;{ }^{13} \mathrm{C}$ $\mathrm{NMR}\left(\mathrm{CDCl}_{3}, 75 \mathrm{MHz}\right) \delta 31.1,33.7,46.7,74.0,85.6,126.5,126.6,127.5,128.2,128.3,129.4,131.2$, 134.1, 134.9, 142.0; EI-MS ( $m / z$, relative intensity): $286\left(\mathrm{M}^{+}, 3\right), 271$ (8), 161 (8), 129 (100). Anal. calcd. for $\mathrm{C}_{17} \mathrm{H}_{15} \mathrm{ClS}$ : C, 71.19; H, 5.27. Found: C, 71.15; H, 5.28.

Phenyl 1-(4-bromophenyl)-1-methyl-2-propynyl sulfide 1h.Yield $33 \%$ (Method A); mp: 57-58 ${ }^{\circ} \mathrm{C}$; IR (film) 3295, 1483, 1077, 1009, 828, 749, $692 \mathrm{~cm}^{-1} ;{ }^{1} \mathrm{H}$ NMR $\left(\mathrm{CDCl}_{3}, 300 \mathrm{MHz}\right) \delta 1.91$ (s, 3H), 2.70

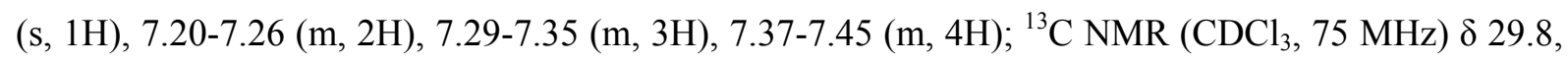
$48.8,75.0,85.5,121.4,128.4,128.5,129.3,131.0,131.8,136.4,141.1$; EI-MS $(\mathrm{m} / \mathrm{z}$, relative intensity): $316\left(\mathrm{M}^{+}, 5\right), 301$ (15), 237 (21), 207 (100), 128 (63). Anal. calcd. for $\mathrm{C}_{16} \mathrm{H}_{13} \mathrm{BrS}$ : C, 60.76; H, 4.11. Found: C, 60.66; H, 4.23.

Ethyl 4-phenyl-4-phenylthio pent-2-ynoate 1i. Yield 20 \% (Method A); IR (film) 2983, 1709, 1257, 1216, 1028, 750, $693 \mathrm{~cm}^{-1}$; ${ }^{1} \mathrm{H}$ NMR $\left(\mathrm{CDCl}_{3}, 300 \mathrm{MHz}\right) \delta 1.34(\mathrm{t}, J=7.2 \mathrm{~Hz}, 3 \mathrm{H}), 1.95(\mathrm{~s}, 3 \mathrm{H}), 4.26$ $(\mathrm{q}, J=7.2 \mathrm{~Hz}, 2 \mathrm{H}), 7.22-7.41(\mathrm{~m}, 8 \mathrm{H}), 7.51-7.54(\mathrm{~m}, 2 \mathrm{H}) ;{ }^{13} \mathrm{C} \mathrm{NMR}\left(\mathrm{CDCl}_{3}, 75 \mathrm{MHz}\right) \delta 14.1,29.0$, 49.0, 61.9, 78.4, 89.1, 126.6, 127.8, 128.2, 128.5, 129.6, 131.4, 136.8, 140.5, 153.6; EI-MS (m/z, relative intensity): $310\left(\mathrm{M}^{+}, 12\right), 264$ (29), 237 (14), 201 (51), 129 (100). Anal. calcd. for $\mathrm{C}_{19} \mathrm{H}_{18} \mathrm{O}_{2} \mathrm{~S}$ : C, 73.52; H, 5.84. Found: C, 73.52; H, 5.88.

Phenyl 1-phenyl-2-propynyl sulfide 1j. ${ }^{15}$ Yield $20 \%$ (Method B); IR (film) 3292, 1481, 1439, 747, $691 \mathrm{~cm}^{-1} ;{ }^{1} \mathrm{H}$ NMR $\left(\mathrm{CDCl}_{3}, 300 \mathrm{MHz}\right) \delta 2.57$ (d, $\left.J=2.4 \mathrm{~Hz}, 1 \mathrm{H}\right), 4.99$ (d, $\left.J=2.4 \mathrm{~Hz}, 1 \mathrm{H}\right), 72.6-7.32$ 
(m, 6H), 7.38-7.43 (m, 4H); ${ }^{13} \mathrm{C} \mathrm{NMR}\left(\mathrm{CDCl}_{3}, 75 \mathrm{MHz}\right) \delta 43.1,74.6,81.9,127.9,127.9,128.3,128.4$, 128.7, 133.2, 133.8, 137.3; EI-MS ( $m / z$, relative intensity): $224\left(\mathrm{M}^{+}, 21\right), 147$ (11), 115 (100).

For the synthesis of $\mathbf{1 g}$, the following procedure was followed:

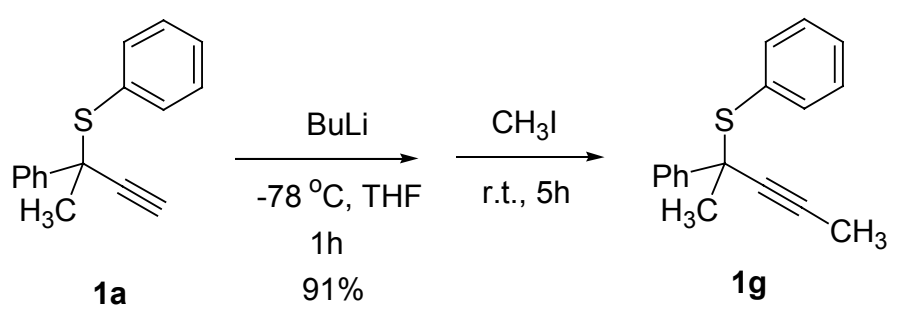

2-Chlorophenylthio-3-methyl indene 2b. IR (film) 3064, 1449, 1032, 750, $717 \mathrm{~cm}^{-1} ;{ }^{1} \mathrm{H}$ NMR $\left(\mathrm{CDCl}_{3}, 300 \mathrm{MHz}\right) \delta 2.27(\mathrm{t}, J=2.1 \mathrm{~Hz}, 3 \mathrm{H}), 3.49(\mathrm{~d}, J=2.1 \mathrm{~Hz}, 2 \mathrm{H}), 6.98-7.01(\mathrm{~m}, 1 \mathrm{H}), 7.08-7.11$ $(\mathrm{m}, 2 \mathrm{H}), 7.25-7.29(\mathrm{~m}, 2 \mathrm{H}), 7.33-7.42(\mathrm{~m}, 3 \mathrm{H}) ;{ }^{13} \mathrm{C} \mathrm{NMR}\left(\mathrm{CDCl}_{3}, 75 \mathrm{MHz}\right) \delta 11.7,42.2,119.6,123.5$, $125.8,126.5,127.1,128.7,129.4,129.7,132.5,136.3,143.6,145.2,147.5$; EI-MS $(\mathrm{m} / \mathrm{z}$, relative intensity): $272\left(\mathrm{M}^{+}, 20\right), 129$ (87), 91 (100). Anal. calcd. for $\mathrm{C}_{16} \mathrm{H}_{13} \mathrm{ClS}$ : C, 70.45; H, 4.80. Found: C, $70.43 ; \mathrm{H}, 4.83$.

3-Methyl-2-naphthylthio indene 2c. IR (film) 3052, 1500, 1459, 851, 814, 757, 743, $717 \mathrm{~cm}^{-1} ;{ }^{1} \mathrm{H}$ NMR $\left(\mathrm{CDCl}_{3}, 300 \mathrm{MHz}\right) \delta 2.31(\mathrm{t}, J=2.1 \mathrm{~Hz}, 3 \mathrm{H}), 3.47(\mathrm{~d}, J=2.1 \mathrm{~Hz}, 2 \mathrm{H}), 7.19-7.25(\mathrm{~m}, 1 \mathrm{H}), 7.32-$ $7.54(\mathrm{~m}, 6 \mathrm{H}), 7.70-7.80(\mathrm{~m}, 4 \mathrm{H}) ;{ }^{13} \mathrm{C} \mathrm{NMR}\left(\mathrm{CDCl}_{3}, 75 \mathrm{MHz}\right) \delta 11.7,42.3,119.2,123.3,125.3,125.8$, 126.5, 126.6, 127.2, 127.5, 127.7, 128.6, 131.9, 132.1, 133.8, 143.3, 143.9, 145.6; EI-MS ( $\mathrm{m} / \mathrm{z}$, relative intensity): 288 ( $\left.\mathrm{M}^{+}, 59\right), 273$ (10), 160 (21), 129 (100), 115 (18). Anal. calcd. for $\mathrm{C}_{20} \mathrm{H}_{16} \mathrm{~S}: \mathrm{C}, 83.29$; $\mathrm{H}$, 5.59. Found: C, 82.87; H, 5.46.

2-Benzylthio-3-methyl- indene 2d. IR (film) 3062, 3028, 1460, 756, 715, $697 \mathrm{~cm}^{-1} ;{ }^{1} \mathrm{H} \mathrm{NMR}\left(\mathrm{CDCl}_{3}\right.$, $300 \mathrm{MHz}) \delta 2.06(\mathrm{t}, J=2.1 \mathrm{~Hz}, 3 \mathrm{H}), 3.43(\mathrm{~d}, J=2.1 \mathrm{~Hz}, 2 \mathrm{H}), 4.00(\mathrm{~s}, 2 \mathrm{H}), 7.09-7.42(\mathrm{~m}, 9 \mathrm{H}) ;{ }^{13} \mathrm{C}$ $\mathrm{NMR}\left(\mathrm{CDCl}_{3}, 75 \mathrm{MHz}\right) \delta 11.2,38.2,41.8,118.5,123.0,124.5,126.4,127.1,128.5,128.6,133.7$, 138.3, 140.2, 142.7, 145.9; EI-MS (m/z, relative intensity): 252 ( $\left.\mathrm{M}^{+}, 60\right), 161$ (55), 128 (53), 91 (100). Anal. calcd. for $\mathrm{C}_{17} \mathrm{H}_{16} \mathrm{~S}$ : C, 80.90; H, 6.39. Found: C, 80.59; H, 6.38 .

3-Methyl-2-hexylthio indene 2e. IR (film) 2927, 1463, 756, $717 \mathrm{~cm}^{-1} ;{ }^{1} \mathrm{H} \mathrm{NMR}\left(\mathrm{CDCl}_{3}, 300 \mathrm{MHz}\right) \delta$ $0.88(\mathrm{t}, J=6.6 \mathrm{~Hz}, 3 \mathrm{H}), 1.28-1.32(\mathrm{~m}, 6 \mathrm{H}), 1.55-1.65(\mathrm{~m}, 2 \mathrm{H}), 2.14(\mathrm{t}, J=1.8 \mathrm{~Hz}, 3 \mathrm{H}), 2.80$ (t, $J=7.2$ $\mathrm{Hz}, 2 \mathrm{H}), 3.48(\mathrm{~d}, J=1.8 \mathrm{~Hz}, 2 \mathrm{H}), 7.11-7.38(\mathrm{~m}, 4 \mathrm{H}) ;{ }^{13} \mathrm{C} \mathrm{NMR}\left(\mathrm{CDCl}_{3}, 75 \mathrm{MHz}\right) \delta 11.3,14.0,22.5$, 28.4, 30.2, 31.4, 33.2, 41.3, 118.1, 122.9, 124.1, 126.4, 134.7, 138.3, 142.5, 146.2; EI-MS (m/z, relative intensity): $246\left(\mathrm{M}^{+}, 62\right), 162(20), 129$ (100). Anal. calcd. for $\mathrm{C}_{16} \mathrm{H}_{22} \mathrm{~S}: \mathrm{C}, 77.99 ; \mathrm{H}, 9.00$. Found: C, 77.89; H, 8.85. 
2-Chlorobenzylthio-3-methyl indene 2f. IR (film) 3065, 1462, 1443, 1052, 1037, 756, 739, $717 \mathrm{~cm}^{-1}$; ${ }^{1} \mathrm{H} \mathrm{NMR}\left(\mathrm{CDCl}_{3}, 300 \mathrm{MHz}\right) \delta 2.02(\mathrm{t}, J=2.1 \mathrm{~Hz}, 3 \mathrm{H}), 3.47$ (d, $\left.J=2.1 \mathrm{~Hz}, 2 \mathrm{H}\right), 4.10(\mathrm{~s}, 2 \mathrm{H}), 7.10-$ $7.38(\mathrm{~m}, 8 \mathrm{H}) ;{ }^{13} \mathrm{C} \mathrm{NMR}\left(\mathrm{CDCl}_{3}, 75 \mathrm{MHz}\right) \delta 11.2,35.9,42.1,118.7,123.1,124.7,126.4,126.8,128.4$, 129.6, 130.5, 132.9, 133.9, 135.9, 141.8, 142.8, 145.7; EI-MS ( $\mathrm{m} / \mathrm{z}$, relative intensity): $286\left(\mathrm{M}^{+}, 50\right)$, 251 (8), 161 (100), 128 (76). Anal. calcd. for $\mathrm{C}_{17} \mathrm{H}_{15} \mathrm{ClS}$ : C, 71.19; H, 5.27. Found: C, 70.97; H, 5.21.

The structure of $\mathbf{2 g}$ was further confirmed by X-ray analysis after being oxidized to its corresponding sulfone 18.
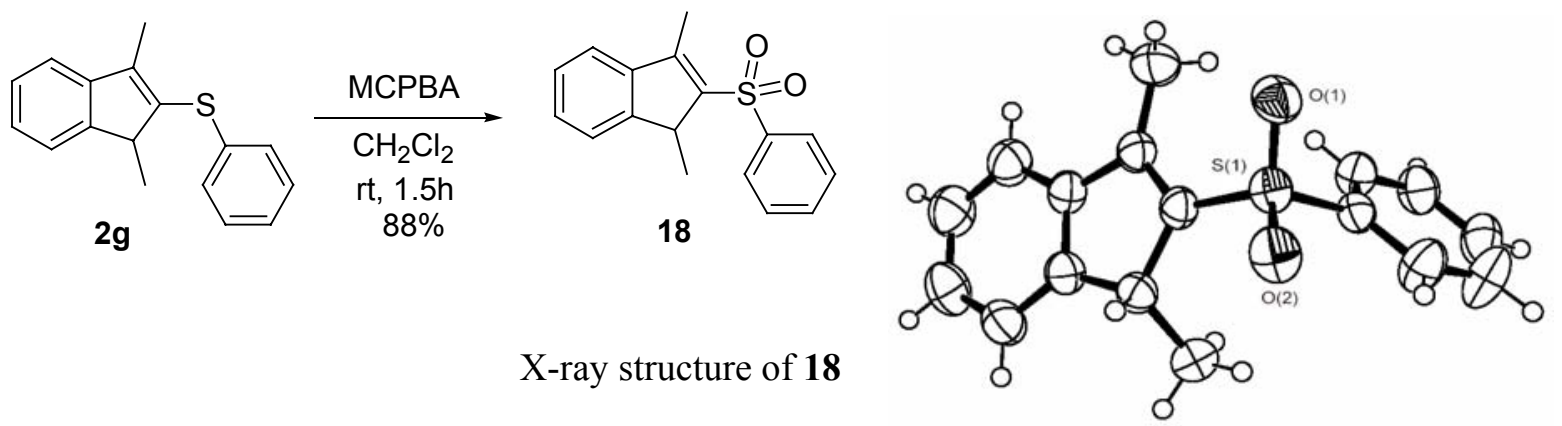

6-Bromo-3-methyl-2-phenylthio indene 2h. IR (film) 3057, 1581, 1459, 872, 814, 741, $690 \mathrm{~cm}^{-1} ;{ }^{1} \mathrm{H}$ NMR $\left(\mathrm{CDCl}_{3}, 300 \mathrm{MHz}\right) \delta 2.23(\mathrm{t}, J=2.1 \mathrm{~Hz}, 3 \mathrm{H}), 3.39(\mathrm{~d}, J=2.1 \mathrm{~Hz}, 2 \mathrm{H}), 7.16-7.30(\mathrm{~m}, 6 \mathrm{H}), 7.43-$ $7.46(\mathrm{~m}, 2 \mathrm{H}) ;{ }^{13} \mathrm{C} \mathrm{NMR}\left(\mathrm{CDCl}_{3}, 75 \mathrm{MHz}\right) \delta 11.6,41.8,119.3,120.2,126.5,126.6,129.1,129.5,129.8$, 133.0, 135.7, 142.6, 144.5, 145.0; EI-MS ( $m / z$, relative intensity): $316\left(\mathrm{M}^{+}, 60\right), 237(10), 207$ (95), 128 (100). Anal. calcd. for $\mathrm{C}_{17} \mathrm{H}_{15} \mathrm{ClS}$ : C, 60.58; H, 4.13. Found: C, 60.58; H, 4.09.

Ethyl 3-methyl-2-phenylthio indene-1-carboxylate 2i. IR (film) 3063, 1733, 1302, 1189, 1142, 1024, $740,691 \mathrm{~cm}^{-1} ;{ }^{1} \mathrm{H}$ NMR $\left(\mathrm{CDCl}_{3}, 300 \mathrm{MHz}\right) \delta 1.18(\mathrm{~d}, J=7.2 \mathrm{~Hz}, 3 \mathrm{H}), 2.26(\mathrm{~d}, J=1.8 \mathrm{~Hz}, 3 \mathrm{H}), 4.00-$ $4.16(\mathrm{~m}, 2 \mathrm{H}), 4.22(\mathrm{~d}, J=1.8 \mathrm{~Hz}, 1 \mathrm{H}), 7.16-7.41(\mathrm{~m}, 8 \mathrm{H}), 7.44-7.46(\mathrm{~m}, 1 \mathrm{H}) ;{ }^{13} \mathrm{C} \mathrm{NMR}\left(\mathrm{CDCl}_{3}, 75\right.$ $\mathrm{MHz}) \delta 11.8,14.0,57.9,61.1,119.6,123.1,126.3,127.8,128.9,129.2,131.0,133.9,135.7,141.4$, 144.8, 147.5, 169.9; EI-MS ( $m / z$, relative intensity): $310\left(\mathrm{M}^{+}, 100\right), 237$ (30), 222 (28), 128 (46). Anal. calcd. for $\mathrm{C}_{19} \mathrm{H}_{18} \mathrm{O}_{2} \mathrm{~S}$ : C, 73.52; H, 5.84. Found: $\mathrm{C}, 73.53 ; \mathrm{H}, 5.67$. 

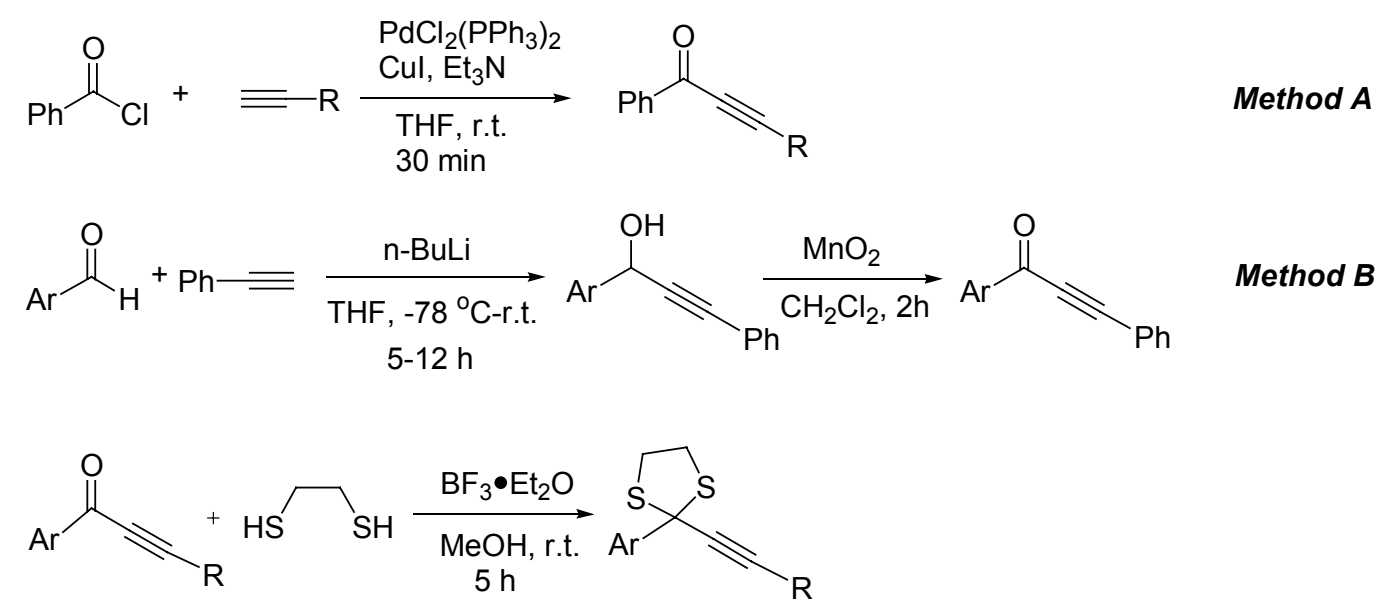

2-(4-Fluolophenyl)-2-(2-phenylethynyl)-1,3-dithiolane 13c. Yield $18 \%$ (white solid); IR (film) 1599, 1503, 1231, 1158, 842, 755, $690 \mathrm{~cm}^{-1} ;{ }^{1} \mathrm{H}$ NMR $\left(\mathrm{CDCl}_{3}, 300 \mathrm{MHz}\right) \delta 3.66-3.80$ (m, 4H), 7.00$7.08(\mathrm{~m}, 2 \mathrm{H}), 7.32-7.34(\mathrm{~m}, 3 \mathrm{H}), 7.48-7.52(\mathrm{~m}, 2 \mathrm{H}), 7.97-8.02(\mathrm{~m}, 2 \mathrm{H}) ;{ }^{13} \mathrm{C} \mathrm{NMR}\left(\mathrm{CDCl}_{3}, 75 \mathrm{MHz}\right) \delta$ 41.3, 61.7, 87.0, 90.9, 114.9, 115.2, 122.5, 128.2, 128.5, 129.5, 129.7, 131.6, 134.4, 134.5, 160.9, 164.1; EI-MS ( $\mathrm{m} / \mathrm{z}$, relative intensity): 300 (M+ $\left.\mathrm{M}^{+}, 78\right), 272$ (68), 239 (100), 207 (56), 163 (20), 145 (41). Anal. calcd for $\mathrm{C}_{17} \mathrm{H}_{13} \mathrm{FS}_{2}$ : C, 67.97; H, 4.36. Found: C, 67.93; H, 4.37.

2-(3-Bromophenyl)-2-(2-phenylethynyl)-1,3-dithiolane 13d.Yield $47 \%$ (white solid); mp: $102{ }^{\circ} \mathrm{C}$; IR (film) 1589, 1565, 1489, 1442, 1416, 755, $690 \mathrm{~cm}^{-1} ;{ }^{1} \mathrm{H}$ NMR $\left(\mathrm{CDCl}_{3}, 300 \mathrm{MHz}\right) \delta 3.65-3.79$ (m, $4 \mathrm{H}), 7.20-7.52(\mathrm{~m}, 7 \mathrm{H}), 7.93-7.96(\mathrm{~m}, 1 \mathrm{H}), 8.16-8.17(\mathrm{~m}, 1 \mathrm{H}) ;{ }^{13} \mathrm{C} \mathrm{NMR}\left(\mathrm{CDCl}_{3}, 75 \mathrm{MHz}\right) \delta 41.5$, $61.4,87.3,90.3,122.2,122.4,126.4,128.2,128.6,129.7,130.7,131.4,131.6,141.5$; EI-MS $(\mathrm{m} / z$, relative intensity): 360 (M+27), 332 (17), 299 (20), 281 (79), 268 (5), 253 (100), 221 (58), 189 (50), 145 (64). Anal. calcd for $\mathrm{C}_{17} \mathrm{H}_{13} \mathrm{BrS}_{2}$ : C, 56.51; H, 3.63. Found: C, 56.23, 3.66.

2-(4-Phenylphenyl)-2-(2-phenylethynyl)-1,3-dithiolane 13e. Yield $43 \%$ (white solid); mp: 97-98 ${ }^{\circ} \mathrm{C}$; IR (film) 1598, 1486, 908, 852, 746, $691 \mathrm{~cm}^{-1} ;{ }^{1} \mathrm{H}$ NMR $\left(\mathrm{CDCl}_{3}, 300 \mathrm{MHz}\right) \delta 3.61-3.76(\mathrm{~m}, 4 \mathrm{H})$, 7.28-7.59 (m, 12H), 8.08-8.10 (m, 2H); ${ }^{13} \mathrm{C} \mathrm{NMR}\left(\mathrm{CDCl}_{3}, 75 \mathrm{MHz}\right) \delta 41.3,62.0,86.8,91.0,122.6$, 126.9, 127.0, 127.4, 128.0, 128.2, 128.4, 128.7, 131.5, 137.8, 140.3, 141.1; EI-MS ( $/ \mathrm{z} / \mathrm{z}$, relative intensity): 358 ( $\left.\mathrm{M}^{+}, 100\right), 330$ (56), 297 (77), 265 (24), 253 (16), 221 (17), 145 (25). HRMS calcd for $\mathrm{C}_{23} \mathrm{H}_{18} \mathrm{~S}_{2}[\mathrm{M}] 358.0846$. Found 358.0850.

2-(Hex-1-yn-1-yl)-2-phenthyl-1,3-ditholane 13f. ${ }^{11 a}$ Yield 36 \% (oil); IR (film) 2956, 2928, 1596, 1446, 717, $693 \mathrm{~cm}^{-1}$; ${ }^{1} \mathrm{H}$ NMR $\left(\mathrm{CDCl}_{3}, 300 \mathrm{MHz}\right) \delta 0.93$ (t, $\left.J=7.2 \mathrm{~Hz}, 3 \mathrm{H}\right), 1.41-1.49$ (m, 2H), 1.51$1.62(\mathrm{~m}, 2 \mathrm{H}), 2.36(\mathrm{t}, J=7.2 \mathrm{~Hz}, 2 \mathrm{H}), 3.61-3.75(\mathrm{~m}, 4 \mathrm{H}), 7.25-7.38(\mathrm{~m}, 3 \mathrm{H}), 7.94-7.98(\mathrm{~m}, 2 \mathrm{H}) ;{ }^{13} \mathrm{C}$ 
$\mathrm{NMR}\left(\mathrm{CDCl}_{3}, 75 \mathrm{MHz}\right) \delta 13.6,18.8,22.0,30.7,41.1,62.2,82.2,88.0,127.5,128.0,128.1,139.4$; EIMS ( $m / z$, relative intensity): $262\left(\mathrm{M}^{+}, 92\right), 233$ (18), 219 (23), 205 (91), 192 (100), 177 (33), 128 (47), 115 (58), 77 (29).

2-(2-Bromophenyl)-2-(2-phenylethynyl)-1,3-dithiolane 13g. Yield $50 \%$ (white solid); mp: $69{ }^{\circ} \mathrm{C}$; IR (film) 2921, 1489, 1455, 1278, 1026, 908, 756, 735, $690 \mathrm{~cm}^{-1} ;{ }^{1} \mathrm{H}$ NMR $\left(\mathrm{CDCl}_{3}, 300 \mathrm{MHz}\right) 3.35-$ $3.46(\mathrm{~m}, 2 \mathrm{H}), 3.58-3.66(\mathrm{~m}, 2 \mathrm{H}), 7.11-7.16(\mathrm{~m}, 1 \mathrm{H}), 7.26-7.32(\mathrm{~m}, 4 \mathrm{H}), 7.44-7.47$ (m, 2H), $7.68(\mathrm{~d}, J$ $=7.8 \mathrm{~Hz}, 1 \mathrm{H}), 8.13(\mathrm{~d}, J=7.8 \mathrm{~Hz}, 1 \mathrm{H}) ;{ }^{13} \mathrm{C} \mathrm{NMR}\left(\mathrm{CDCl}_{3}, 75 \mathrm{MHz}\right) 40.66,62.72,85.00,89.54,122.60$, $123.78,127.08,127.61,128.03,128.27,129.23,131.54,135.27,139.60$; EI-MS ( $\mathrm{m} / z$, relative intensity): 360 (M+, 39), 332 (13), 299 (19), 281 (54), 253 (100), 221 (74), 189 (66), 145 (35). HRMS calcd for $\mathrm{C}_{17} \mathrm{H}_{13} \mathrm{~S}_{2}{ }^{79} \mathrm{Br}[\mathrm{M}]$ 359.9642. Found 359.9658.

2-(4-Methylphenylethynyl)-2-phenyl -1,3-dithiolane 13h. Yield $52 \%$ (white solid); mp: 58-59 ${ }^{\circ} \mathrm{C}$; IR (film) 2922, 1509, 1446, 1275, 1022, 816, 721, $702 \mathrm{~cm}^{-1}$; ${ }^{1} \mathrm{H}$ NMR ( $\left.\mathrm{CDCl}_{3}, 300 \mathrm{MHz}\right) 2.35$ (s, 3H), 3.66-3.80 (m, 4H), 7.12-7.16 (m, 2H), 7.28-7.41 (m, 5H), 8.01-8.04 (m, 2H); ${ }^{13} \mathrm{C} \mathrm{NMR}\left(\mathrm{CDCl}_{3}, 75\right.$ MHz) 21.46, 41.28, 62.34, 87.02, 90.33, 119.61, 127.62, 128.18, 128.28, 128.95, 131.47, 138.50, 138.83; EI-MS (m/z, relative intensity): 296 ( $\left.\mathrm{M}^{+}, 100\right), 268$ (67), 253 (34), 235 (83), 202 (38), 189 (33), 159 (42), 145 (19), 121 (23). Anal. calcd for $\mathrm{C}_{18} \mathrm{H}_{16} \mathrm{~S}_{2}$ : C, 72.93; H, 5.44. Found: C, 72.76; H, 5.43 .

2-(2-Furanyl)-2-(2-phenylethynyl)-1,3-dithiolane 16. Yield $34 \%$ (oil); IR (film) 1598, 1489, 1150, 1005, 811, 756, $690 \mathrm{~cm}^{-1} ;{ }^{1} \mathrm{H}$ NMR $\left(\mathrm{CDCl}_{3}, 300 \mathrm{MHz}\right) \delta 3.63-3.69(\mathrm{~m}, 4 \mathrm{H}), 6.33(\mathrm{dd}, J=1.8$, $3.3 \mathrm{~Hz}, 1 \mathrm{H}), 6.60(\mathrm{dd}, J=0.9,3.3 \mathrm{~Hz}, 1 \mathrm{H}), 7.29-7.34(\mathrm{~m}, 3 \mathrm{H}), 7.44-7.50(\mathrm{~m}, 3 \mathrm{H}) ;{ }^{13} \mathrm{C} \mathrm{NMR}\left(\mathrm{CDCl}_{3}\right.$, $75 \mathrm{MHz}) \delta 41.2,55.2,85.0,88.1,108.2,110.4,122.2,128.1,128.6,131.7,143.2,152.5$; EI-MS $(\mathrm{m} / z$, relative intensity): $272\left(\mathrm{M}^{+}, 63\right), 244$ (100), 212 (30), 184 (39), 152 (56), 126 (68). Anal. calcd for $\mathrm{C}_{15} \mathrm{H}_{12} \mathrm{OS}_{2}$ : C, 66.14; H, 4.44. Found: C, 66.17; H, 4.48 .

6-Bromo-9-phenyl-2,3-dihydro-1,4-dithiofluorene 14b. White solid; mp: $139{ }^{\circ} \mathrm{C}$; IR (film) 1573 , 1533, 1493, 1453, 908, 814, 734, $698 \mathrm{~cm}^{-1} ;{ }^{1} \mathrm{H}$ NMR $\left(\mathrm{CDCl}_{3}, 300 \mathrm{MHz}\right) \delta 3.23-3.29(\mathrm{~m}, 4 \mathrm{H}), 4.51$ (s, 1H), 6.99-7.09 (m, 3H), 7.22-7.41 (m, 5H); ${ }^{13} \mathrm{C} \mathrm{NMR}\left(\mathrm{CDCl}_{3}, 75 \mathrm{MHz}\right) \delta 26.0,27.2,59.1,117.9$, 118.6, 123.2, 126.3, 127.5, 128.1, 128.8, 130.0, 133.2, 137.9, 141.9, 146.9; EI-MS ( $\mathrm{m} / \mathrm{z}$, relative intensity): 360 (M+100), 332 (28), 299 (18), 281 (35), 253 (55), 221 (37), 189 (9), 176 (19), 126 (16). Anal. calcd for $\mathrm{C}_{17} \mathrm{H}_{13} \mathrm{BrS}_{2}$ : C, 56.51; H, 3.63. Found: C, 56.12, 3.58 .

6-Fluoro-9-phenyl-2,3-dihydro-1,4-dithiofluorene 14c. White solid; mp: $103-105{ }^{\circ} \mathrm{C}$; IR (film) 1597, 1537, 1472, 1264, 1211, 820, 739, $700 \mathrm{~cm}^{-1} ;{ }^{1} \mathrm{H}$ NMR $\left(\mathrm{CDCl}_{3}, 300 \mathrm{MHz}\right) \delta 3.19-3.34(\mathrm{~m}, 4 \mathrm{H})$, 
$4.52(\mathrm{~s}, 1 \mathrm{H}), 6.82-6.86(\mathrm{~m}, 1 \mathrm{H}), 6.93-7.00(\mathrm{~m}, 1 \mathrm{H}), 7.04-7.10(\mathrm{~m}, 3 \mathrm{H}), 7.24-7.34(\mathrm{~m}, 3 \mathrm{H}) ;{ }^{13} \mathrm{C}$ NMR $\left(\mathrm{CDCl}_{3}, 75 \mathrm{MHz}\right) \delta 26.1,27.1,59.2,59.3,111.1,111.4,113.5,113.8,117.2,117.3,123.1,127.5$, 128.1, 128.8, 132.0, 138.3, 138.94, 147.0, 147.1, 159.7, 162.9; EI-MS ( $/ \mathrm{z}$, relative intensity): 300 (M+100), 272 (61), 239 (84), 207 (17), 195 (18), 183 (10), 145 (9), 119 (14). Anal. calcd for $\mathrm{C}_{17} \mathrm{H}_{13} \mathrm{FS}_{2}$ : C, 67.97; H, 4.36. Found: C, 68.17; H, 4.37.

7-Bromo-9-phenyl-2,3-dihydro-1,4-dithiofluorene (14d) and it's isomer (a $4: 1$ mixture). The major isomer 14d: White solid; IR (film) 1593, 1536, 1450, 1265, 777, 737, $701 \mathrm{~cm}^{-1}$; ${ }^{1} \mathrm{H}$ NMR $\left(\mathrm{CDCl}_{3}, 300 \mathrm{MHz}\right) \delta 3.17-3.19(\mathrm{~m}, 1 \mathrm{H}), 3.20-3.30(\mathrm{~m}, 4 \mathrm{H}), 4.49(\mathrm{~s}, 0.25 \mathrm{H}), 4.57$ (s, 1H), 6.93-7.32 (m, 10H); ${ }^{13} \mathrm{C} \mathrm{NMR}\left(\mathrm{CDCl}_{3}, 75 \mathrm{MHz}\right) \delta 26.0,27.3,27.4,58.9,60.7,115.7,118.8,120.0,121.2,122.4$, $124.4,127.2,127.4,127.5,128.1,128.4,128.5,128.5,128.8,129.1,134.7,135.2,137.0,138.2,143.5$, 143.8, 145.7; EI-MS ( $\mathrm{m} / z$, relative intensity): 360 ( $\left.\mathrm{M}^{+}, 100\right), 332$ (18), 299 (24), 282 (78), 253 (88), 221 (99), 189 (12), 176 (22), 104 (23). Anal. calcd for $\mathrm{C}_{17} \mathrm{H}_{13} \mathrm{BrS}_{2}$ : C, 56.51; H, 3.63. Found: C, 56.94; $\mathrm{H}$, 3.64. HRMS calcd for $\mathrm{C}_{17} \mathrm{H}_{13} \mathrm{BrS}_{2}[\mathrm{M}] 359.9645$. Found 359.9642 .

6,9-Diphenyl-2,3-dihydro-1,4-dithiofluorene 14e. White solid; mp: $188-189{ }^{\circ} \mathrm{C}$; IR (film) 1599 , 1531, 1493, 1468, 908, 823, 762, 730, $699 \mathrm{~cm}^{-1} ;{ }^{1} \mathrm{H}$ NMR $\left(\mathrm{CDCl}_{3}, 300 \mathrm{MHz}\right) \delta 3.24-3.30(\mathrm{~m}, 4 \mathrm{H})$, $4.61(\mathrm{~s}, 1 \mathrm{H}), 7.12-7.14(\mathrm{~m}, 2 \mathrm{H}), 7.21-7.39(\mathrm{~m}, 8 \mathrm{H}), 7.50-7.53(\mathrm{~m}, 3 \mathrm{H}) ;{ }^{13} \mathrm{C} \mathrm{NMR}\left(\mathrm{CDCl}_{3}, 75 \mathrm{MHz}\right) \delta$ 26.1, 27.3, 59.4, 116.9, 122.0, 123.6, 126.1, 126.9, 127.0, 127.3, 128.3, 128.6, 128.8, 133.0, 137.9, 138.8, 141.2, 142.3, 145.7; EI-MS (m/z, relative intensity): $358\left(\mathrm{M}^{+}, 100\right), 330$ (29), 297 (38), 265 (4), 253 (17), 221 (7), 148 (2). Anal. calcd for $\mathrm{C}_{23} \mathrm{H}_{18} \mathrm{~S}_{2}$ : C, 77.05; H, 5.06. Found: C, 76.62; H, 4.99. HRMS calcd for $\mathrm{C}_{23} \mathrm{H}_{18} \mathrm{~S}_{2}[\mathrm{M}] 358.0846$. Found 358.0850.

9-Butyl-2,3-dihydro-1,4-dithiofluorene 14f. Oil; IR (film) 2955, 2925, 2856, 1602, 1538, 1463, 756, $693 \mathrm{~cm}^{-1}$; ${ }^{1} \mathrm{H}$ NMR $\left(\mathrm{CDCl}_{3}, 300 \mathrm{MHz}\right) \delta 0.84(\mathrm{t}, J=7.2 \mathrm{~Hz}, 3 \mathrm{H}), 0.92-1.02(\mathrm{~m}, 1 \mathrm{H}), 1.17-1.31$ (m, 3H), 1.73-1.83 (m, 1H), 1.87-1.98 (m, 1H), 3.28-3.29 (m, 4H), 3.52 (t, $J=5.4 \mathrm{~Hz}, 1 \mathrm{H}), 7.09-7.15$ (m, 2H), 7.23-7.33 (m, 2H); ${ }^{13} \mathrm{C} \mathrm{NMR}\left(\mathrm{CDCl}_{3}, 75 \mathrm{MHz}\right) \delta$ 13.9, 22.9, 26.1, 26.6, 27.2, 30.8, 52.7, 116.5, 122.0, 122.5, 124.1, 126.5, 132.1, 143.4, 144.2; EI-MS ( $\mathrm{m} / \mathrm{z}$, relative intensity): $262\left(\mathrm{M}^{+}, 100\right)$, 234 (8), 219 (13), 205 (54), 191 (19), 177 (68), 170 (8). Anal. calcd for $\mathrm{C}_{15} \mathrm{H}_{18} \mathrm{~S}_{2}$ : C, 68.65; H, 6.91. Found: C, 68.91; H, 6.90 .

8-Bromo-9-phenyl-2,3-dihydro-1,4-dithiofluorene 14g. White solid; mp: $128-129{ }^{\circ} \mathrm{C}$; IR (film) 2920, 1520, 1452, 1418, 1221, 1121, 908, 764, 734, $702 \mathrm{~cm}^{-1} ;{ }^{1} \mathrm{H}$ NMR $\left(\mathrm{CDCl}_{3}, 300 \mathrm{MHz}\right) 3.19-3.31$ (m, 4H), $4.51(\mathrm{~s}, 1 \mathrm{H}), 6.87-7.10(\mathrm{~m}, 4 \mathrm{H}), 7.25-7.37(\mathrm{~m}, 4 \mathrm{H}) ;{ }^{13} \mathrm{C} \mathrm{NMR}\left(\mathrm{CDCl}_{3}, 75 \mathrm{MHz}\right) 26.81,26.96$, $59.60,112.04,122.25,124.07,125.89,127.49,128.23,128.77,132.03,135.05,138.50,141.22$, 
147.93; EI-MS (m/z, relative intensity): 360 (M+100), 332 (12), 299 (16), 281 (16), 253 (78), 221 (32), 189 (7), 176 (9), 126 (12), 104 (9). HRMS calcd for $\mathrm{C}_{17} \mathrm{H}_{13} \mathrm{~S}_{2}{ }^{79} \mathrm{Br}[\mathrm{M}]$ 359.9642. Found 359.9642.

9-(4-Methylphenyl)-2,3-dihydro-1,4-dithiofluorene 14h. White solid; mp: $119{ }^{\circ} \mathrm{C}$; IR (film) 2920 , 1537, 1511, 1287, 909, 796, 757, $728 \mathrm{~cm}^{-1} ;{ }^{1} \mathrm{H}$ NMR $\left(\mathrm{CDCl}_{3}, 300 \mathrm{MHz}\right) 2.32$ (s, 3H), 3.23-3.32 (m, 4H), 4.52 (s, 1H), 6.97-7.29 (m, 8H); ${ }^{13} \mathrm{C} \mathrm{NMR}\left(\mathrm{CDCl}_{3}, 75 \mathrm{MHz}\right) 21.15,26.09,27.34,58.94,116.66$, $123.01,123.47,124.79,126.92,128.07,129.44,132.74,135.78,126.85,142.98,145.16$; EI-MS $(\mathrm{m} / \mathrm{z}$, relative intensity): $296\left(\mathrm{M}^{+}, 100\right), 268$ (43), 253 (13), 235 (39), 221 (14), 189 (7), 177 (12), 102 (6). HRMS calcd for $\mathrm{C}_{18} \mathrm{H}_{16} \mathrm{~S}_{2}[\mathrm{M}]$ 296.0693. Found 296.0704.

4-Phenyl-5,6-(ethylenedithiyl)cyclopentadieno[1,2-a]furan 16. White solid; IR (film) 1494, 1452, 1137, 1056, 875, 701, $682 \mathrm{~cm}^{-1} ;{ }^{1} \mathrm{H}$ NMR $\left(\mathrm{CDCl}_{3}, 300 \mathrm{MHz}\right) \delta 3.16-3.29(\mathrm{~m}, 4 \mathrm{H}), 4.28(\mathrm{~s}, 1 \mathrm{H}), 6.36$ $(\mathrm{d}, J=1.8 \mathrm{~Hz}, 1 \mathrm{H}), 7.11-7.14(\mathrm{~m}, 3 \mathrm{H}), 7.26-7.36(\mathrm{~m}, 3 \mathrm{H}) ;{ }^{13} \mathrm{C} \mathrm{NMR}\left(\mathrm{CDCl}_{3}, 75 \mathrm{MHz}\right) \delta 26.1,27.1$, 52.6, 108.2, 116.0, 127.0, 127.3, 127.9, 128.7, 132.9, 137.8, 143.8, 159.8; EI-MS $(\mathrm{m} / \mathrm{z}$, relative intensity): $272\left(\mathrm{M}^{+}, 100\right), 244$ (34), 211(16), 171 (19), 139 (12). HRMS calcd for $\mathrm{C}_{15} \mathrm{H}_{12} \mathrm{OS}_{2}$ [M] 272.0330. Found 272.0330.

\section{Mechanistic Study}

Preparation of sulfide $4^{19}$

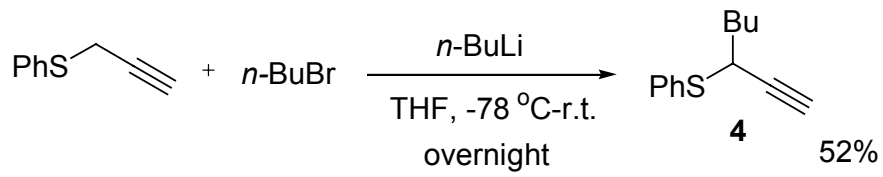

Phenyl 1-butyl-2-propynyl sulfide 4. ${ }^{\text {4a }}$ Oil; IR (film) 3295, 2957, 2931, 1701, 1584, 1439, 1025, 744, $690 \mathrm{~cm}^{-1} ;{ }^{1} \mathrm{H}$ NMR $\left(\mathrm{CDCl}_{3}, 300 \mathrm{MHz}\right) \delta 0.90(\mathrm{t}, J=7.2 \mathrm{~Hz}, 3 \mathrm{H}), 1.27-1.42(\mathrm{~m}, 2 \mathrm{H}), 1.45-1.64(\mathrm{~m}$, 2H), 1.68-1.81 (m, 2H), $2.32(\mathrm{~d}, J=2.4 \mathrm{~Hz}, 1 \mathrm{H}), 3.77(\mathrm{dt}, J=7.2,2.4 \mathrm{~Hz}, 1 \mathrm{H}), 7.23-7.34(\mathrm{~m}, 3 \mathrm{H})$, 7.50-7.54 (m, 2H); ${ }^{13} \mathrm{C} \mathrm{NMR}\left(\mathrm{CDCl}_{3}, 75 \mathrm{MHz}\right) \delta 13.85,22.10,29.14,34.57,38.29,72.14,83.75$, 127.53, 128.73, 132.52, 133.88; EI-MS ( $\mathrm{m} / \mathrm{z}$, relative intensity): $204\left(\mathrm{M}^{+}, 33\right), 161$ (65), 147 (45), 110 (100), 95 (9).

\section{$\mathrm{PtCl}_{2}$-Catalyzed reaction of 4 in the presence of styrene}<smiles>C#CC(CBr)Sc1ccccc1</smiles> 


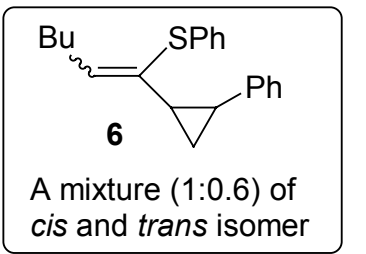

Oil; IR (film) 2956, 2927, 1583, 1477, 1439, 1025, 738, $696 \mathrm{~cm}^{-1} ;{ }^{1} \mathrm{H}$ NMR $\left(\mathrm{CDCl}_{3}, 300 \mathrm{MHz}\right) \delta$ 0.76-0.83 (m, 3H), 0.86-0.90 (m, 3H), 1.01-1.16 (m, $4 \mathrm{H}), 1.22-1.39(\mathrm{~m}, 5 \mathrm{H}), 1.80-1.86(\mathrm{~m}, 1 \mathrm{H}), 1.97-2.04(\mathrm{~m}, 1 \mathrm{H}), 2.12-2.24(\mathrm{~m}$, $1 \mathrm{H}), 2.30-2.37(\mathrm{~m}, 1 \mathrm{H}), 5.72(\mathrm{t}, J=7.2 \mathrm{~Hz}, 0.6 \mathrm{H}), 6.06(\mathrm{t}, J=7.2 \mathrm{~Hz}, 1 \mathrm{H})$, 6.89-6.92 (m, 3H), 7.08-7.28 (m, 13H); ${ }^{13} \mathrm{C} \mathrm{NMR}\left(\mathrm{CDCl}_{3}, 75 \mathrm{MHz}\right) \delta 10.74$, $125.72,125.81,127.50,128.14,128.32,128.65,128.69,128.76,129.03,129.26,129.61,132.59$, 136.31, 137.00, 137.56, 138.06, 142.26; EI-MS ( $m / z$, relative intensity): $308\left(\mathrm{M}^{+}, 47\right), 265$ (7), 251 (5), 199 (15), 156 (69), 141 (36), 128 (33), 115 (29), 109 (57), 91 (100). HRMS calcd for $\mathrm{C}_{21} \mathrm{H}_{24} \mathrm{~S}$ [M] 308.1599. Found 308.1599.

\section{Kinetic Isotope Effect Study}

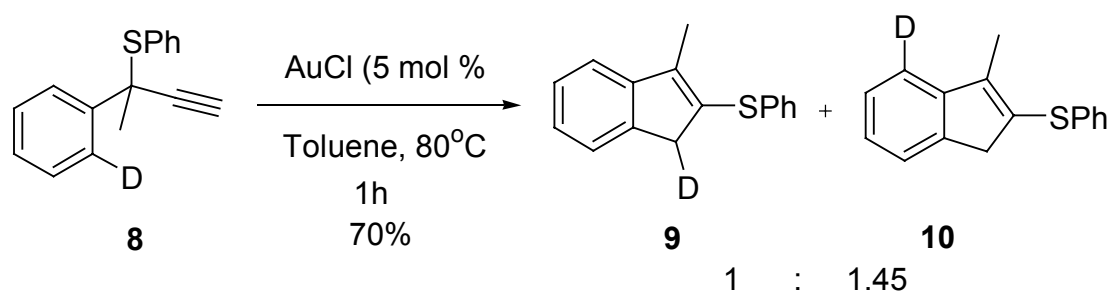

Phenyl 1-methyl-1-(2-d-phenyl)-2-propynyl sulfide 8. White solid; IR (film) 3293, 1473, 1438, 1068, 1026, 774, 750, $692 \mathrm{~cm}^{-1} ;{ }^{1} \mathrm{H}$ NMR $\left(\mathrm{CDCl}_{3}, 300 \mathrm{MHz}\right) \delta 1.94(\mathrm{~s}, 3 \mathrm{H}), 2.69$ (s, 1H), 7.18-7.36 (m, $8 \mathrm{H}), 7.57-7.60(\mathrm{~m}, 1 \mathrm{H}) ;{ }^{13} \mathrm{C} \mathrm{NMR}\left(\mathrm{CDCl}_{3}, 75 \mathrm{MHz}\right) \delta 29.89,49.17,74.64,86.03,126.64,127.41$, 127.88, 128.00, 128.22, 129.09, 132.23, 136.39, 141.71; EI-MS ( $\mathrm{m} / z$, relative intensity): $239\left(\mathrm{M}^{+}, 35\right)$, 130 (100), 109 (16), 77(13).

Mixture of 9 and 10. Oil; IR (film) 3060, 1582, 1475, 1439, 1024, 753, 739, $691 \mathrm{~cm}^{-1}$; ${ }^{1} \mathrm{H}$ NMR $\left(\mathrm{CDCl}_{3}, 400 \mathrm{MHz}\right) \delta 2.26-2.27$ (m, 3H), 3.41-3.44 (m, 1.6H), 7.18-7.35 (m, 9H); ${ }^{13} \mathrm{C} \mathrm{NMR}\left(\mathrm{CDCl}_{3}, 50\right.$ MHz) $\delta 11.65,42.07,119.13,123.30,125.25,126.18,126.34,126.46,128.97,129.35,131.96,132.26$, 136.39, 143.22; EI-MS ( $\mathrm{m} / \mathrm{z}$, relative intensity): $239\left(\mathrm{M}^{+}, 72\right), 162$ (4), 130 (100). HRMS calcd for $\mathrm{C}_{16} \mathrm{H}_{13} \mathrm{SD}[\mathrm{M}] 239.0879$. Found 239.0881.

\section{References}

(4a) Kim, J. T.; Kel'in, A.V.; Gevorgyan, V. Angew. Chem. Int. Ed. 2003, 42, 98.

(11a) Lee, C. -F.; Yang, L.-M.; Hwu, T. -Y.; Feng, A.-S.; Tseng, J.-C.; Luh, T.-Y. J. Am. Chem. Soc. 2000, 122, 4992.

(15) Inada, Y.; Nishibayashi, Y.; Hidai, M.; Uemura, S. J. Am. Chem. Soc. 2002, 124, 15172. 


\section{2-(4-Phenylphenyl)-2-(2-phenylethynyl)-1,3-dithiolane $13 e$}

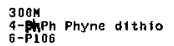

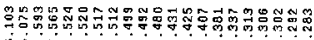

borntisnom

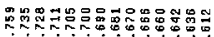

s.s.s $x_{\text {O }}$

1L2401011
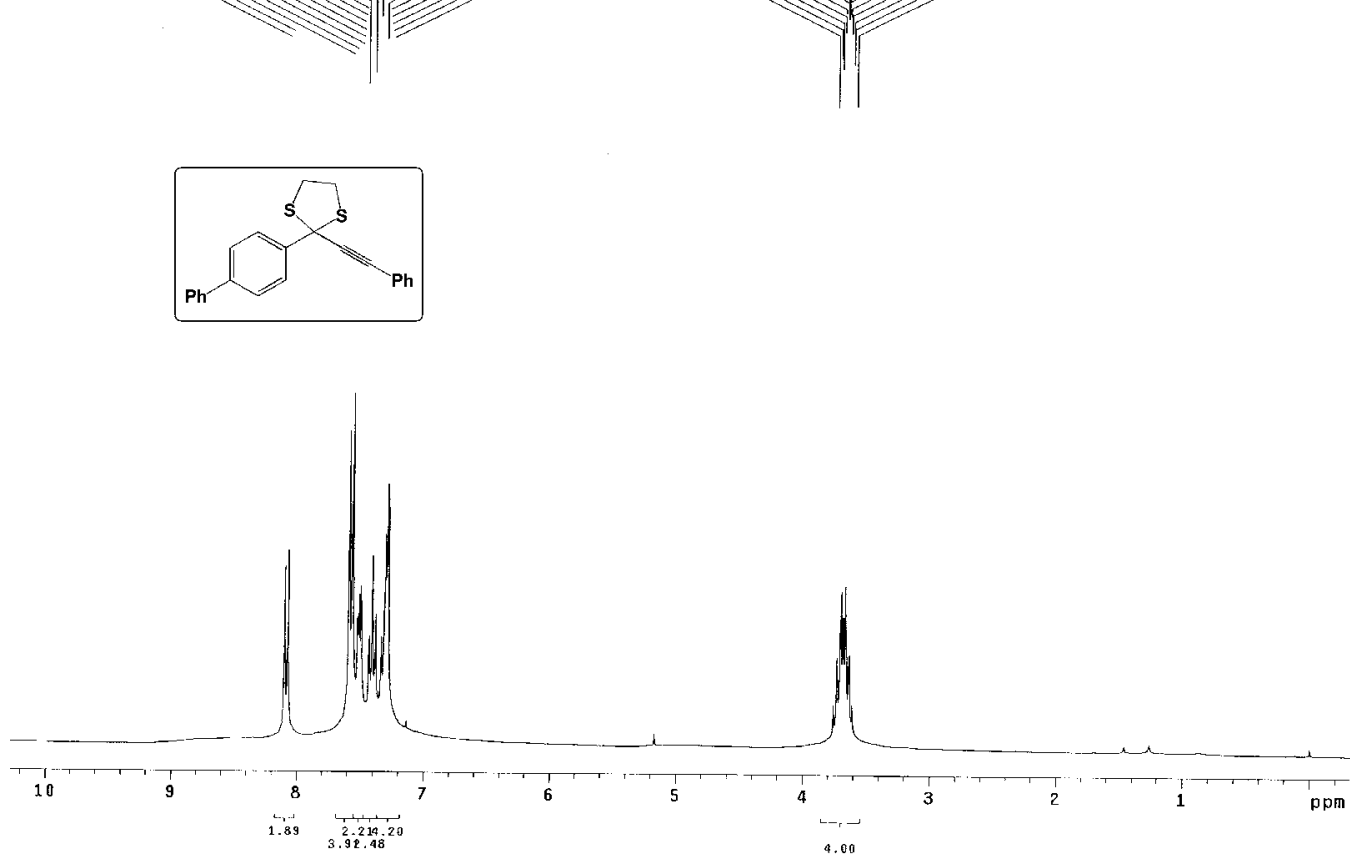

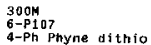
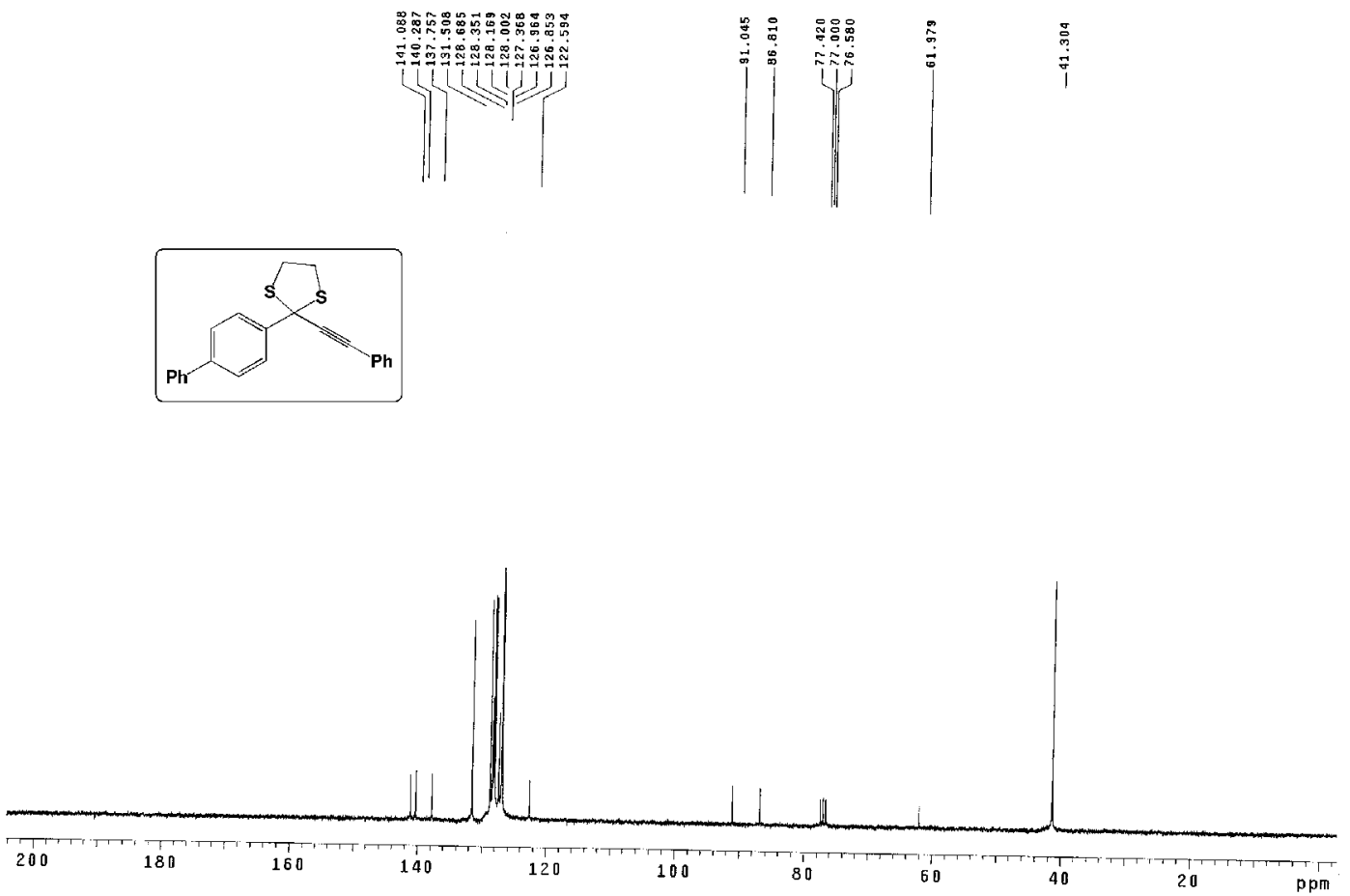
6,9-Diphenyl-2,3-dihydro-1,4-dithiofluorene 14e

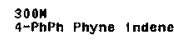
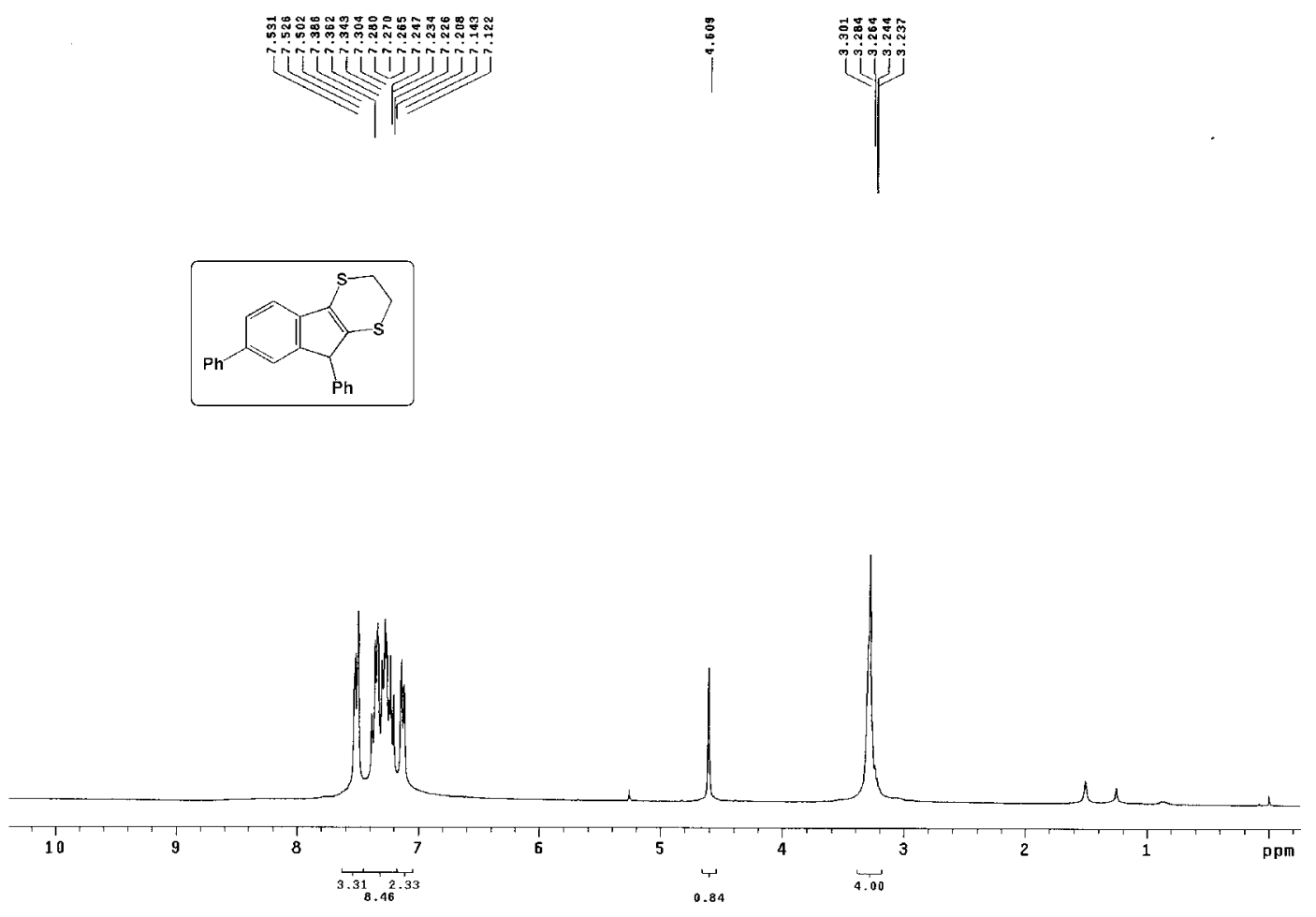

soon 4 s-phph Phyne indene
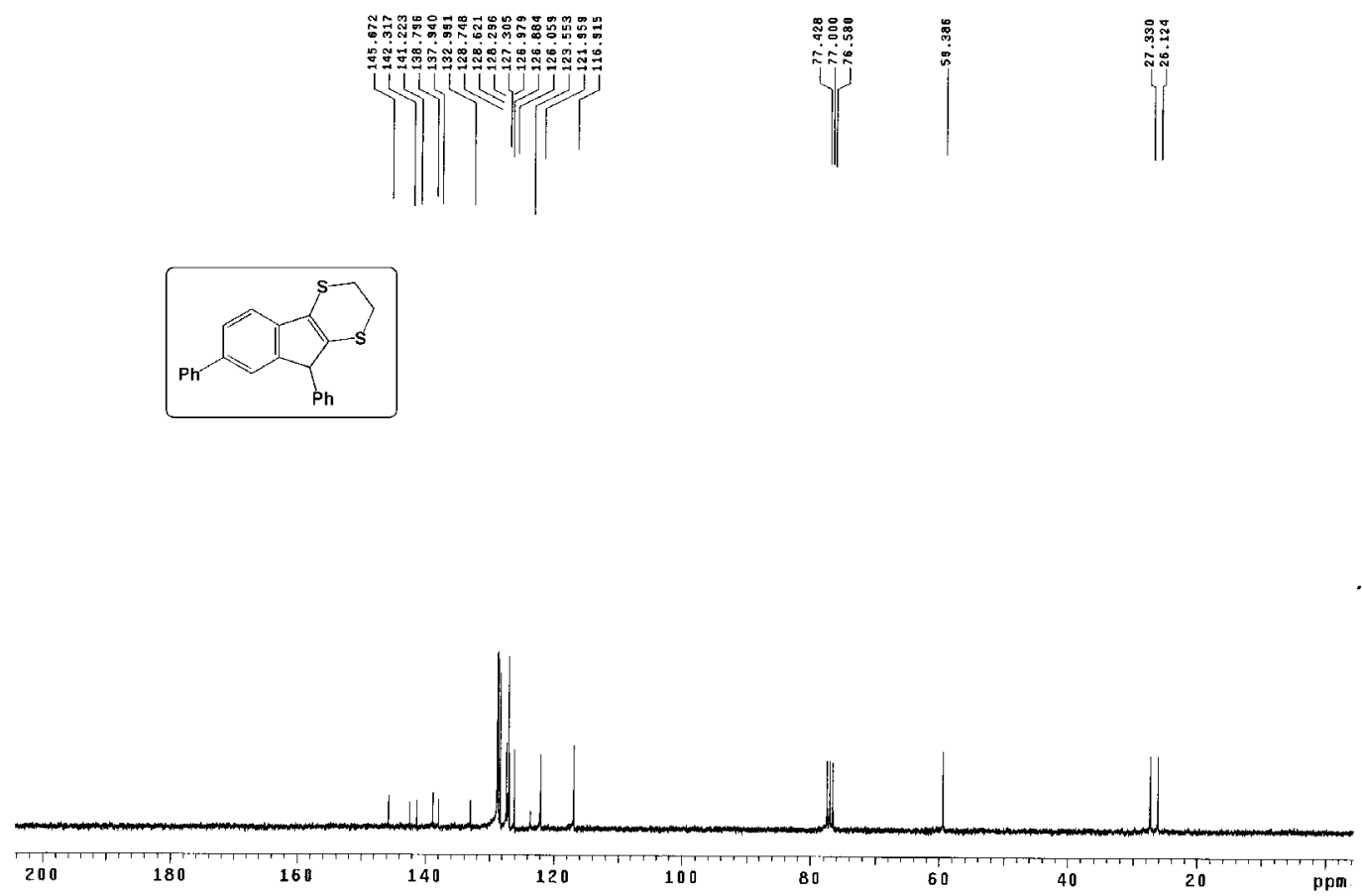
7-Bromo-9-phenyl-2,3-dihydro-1,4-dithiofluorene (14d) and it's isomer (a $4: 1$ mixture)

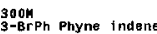
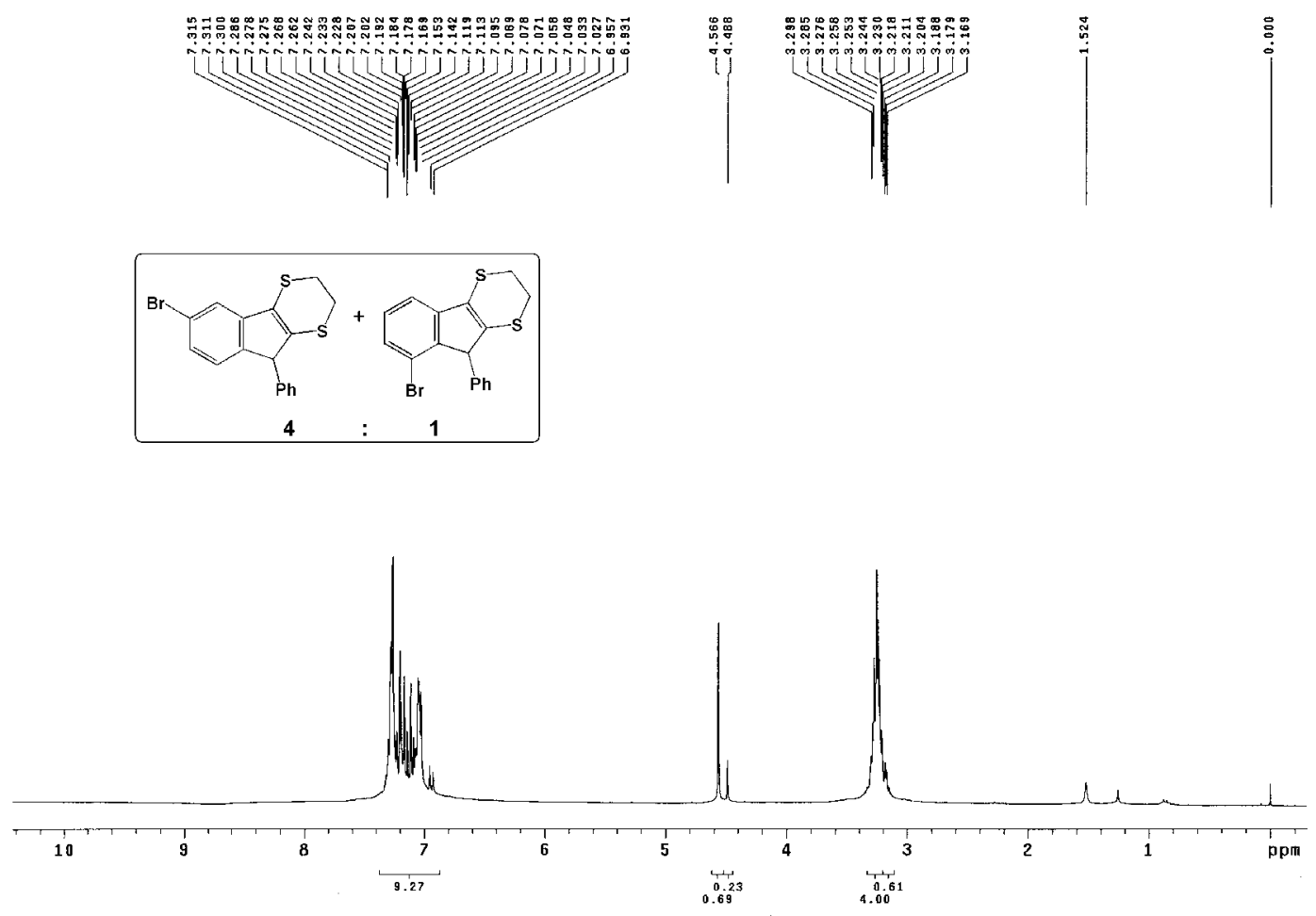

${ }_{3-B r P h}^{300 M}$ Phyne indene
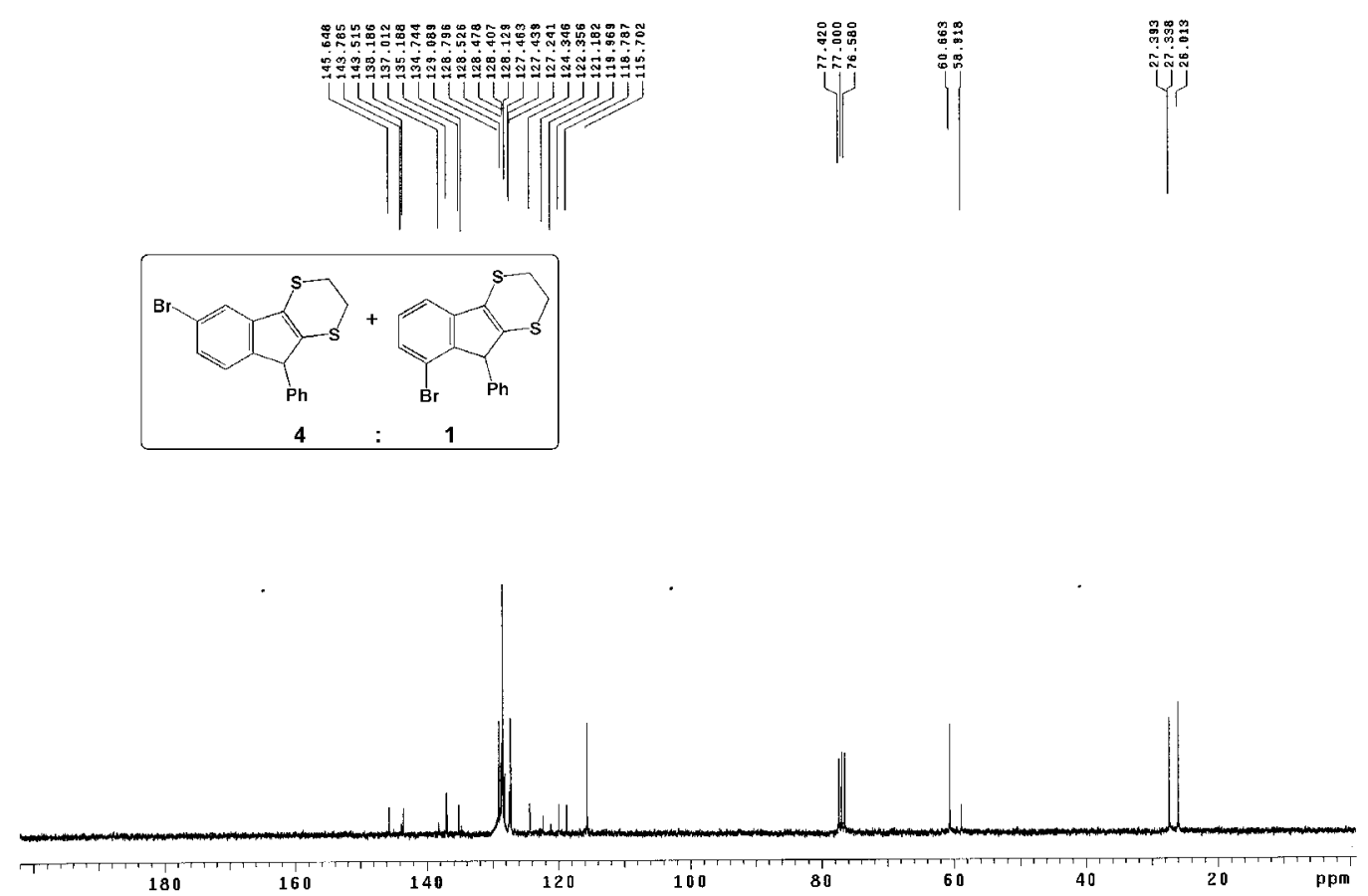


\section{8-Bromo-9-phenyl-2,3-dihydro-1,4-dithiofluorene 13g}

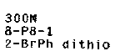

Archive diroctory: /export/home/vnir. 1/vamrsys/data
Sample directory:

Pulse Sequence: s2pu

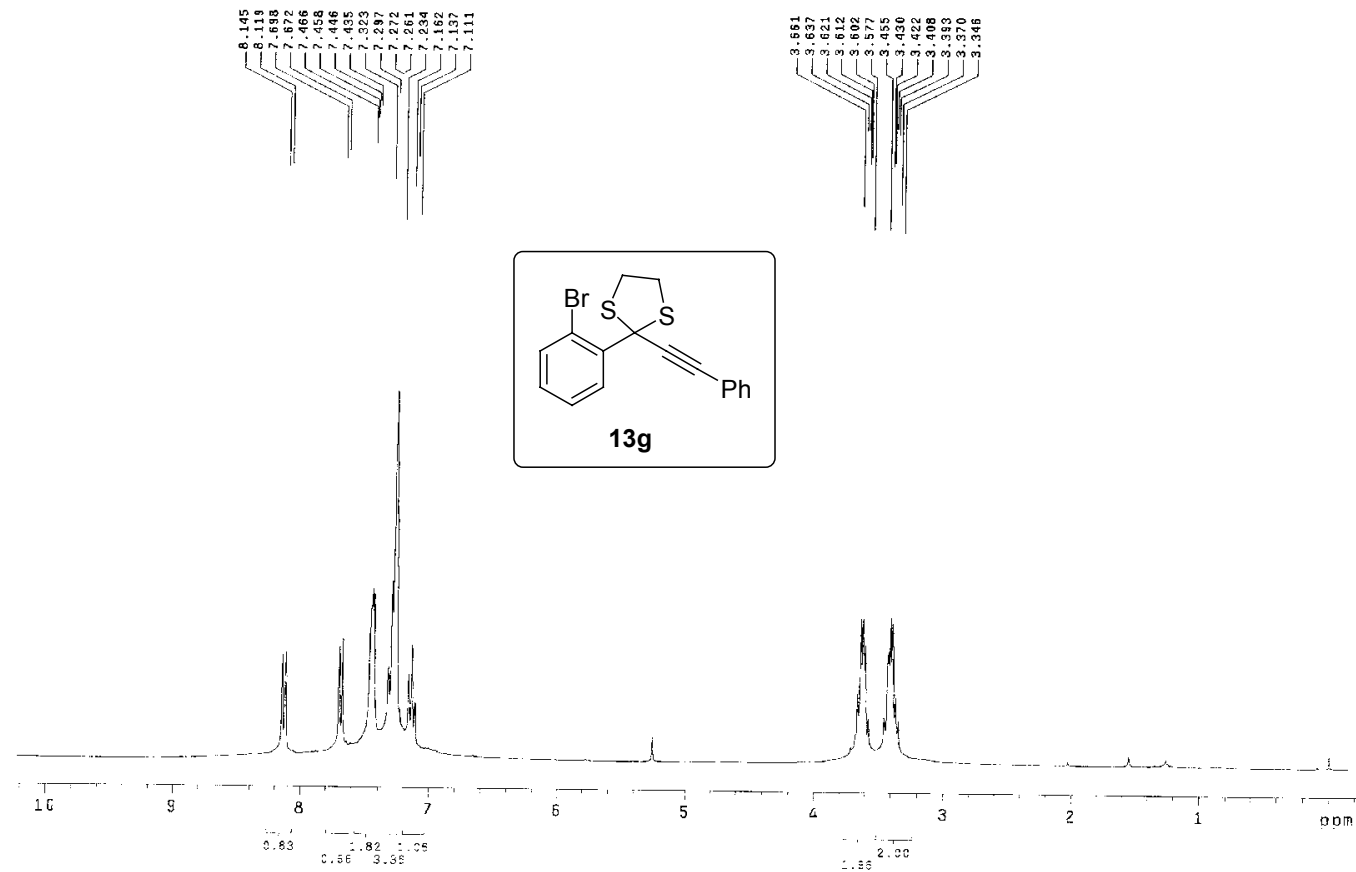

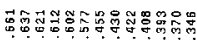

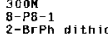

Pulse Sequence: s2pul
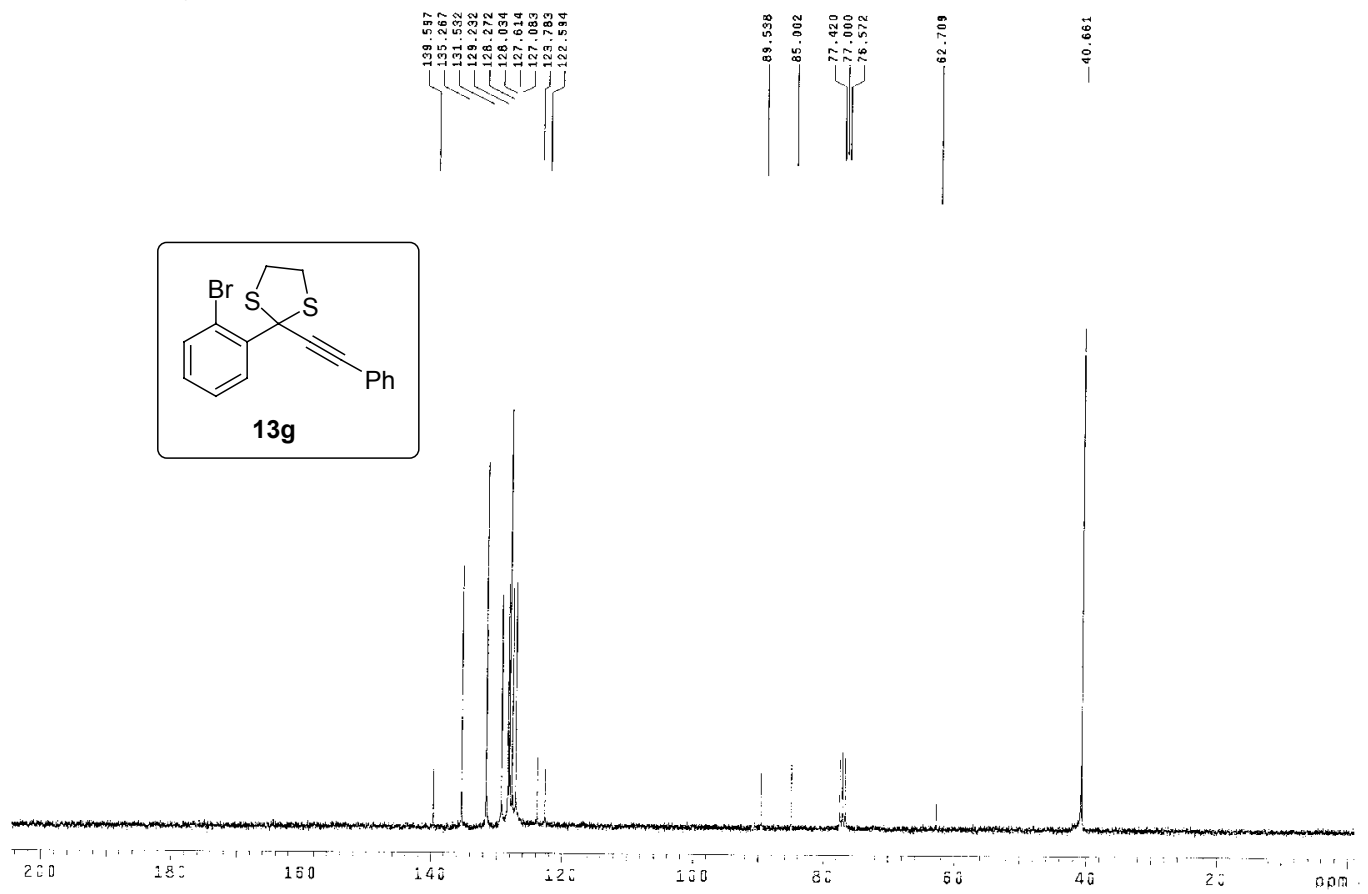


\section{8-Bromo-9-phenyl-2,3-dihydro-1,4-dithiofluorene 14g}

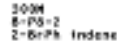

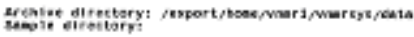

notse sequencti spest
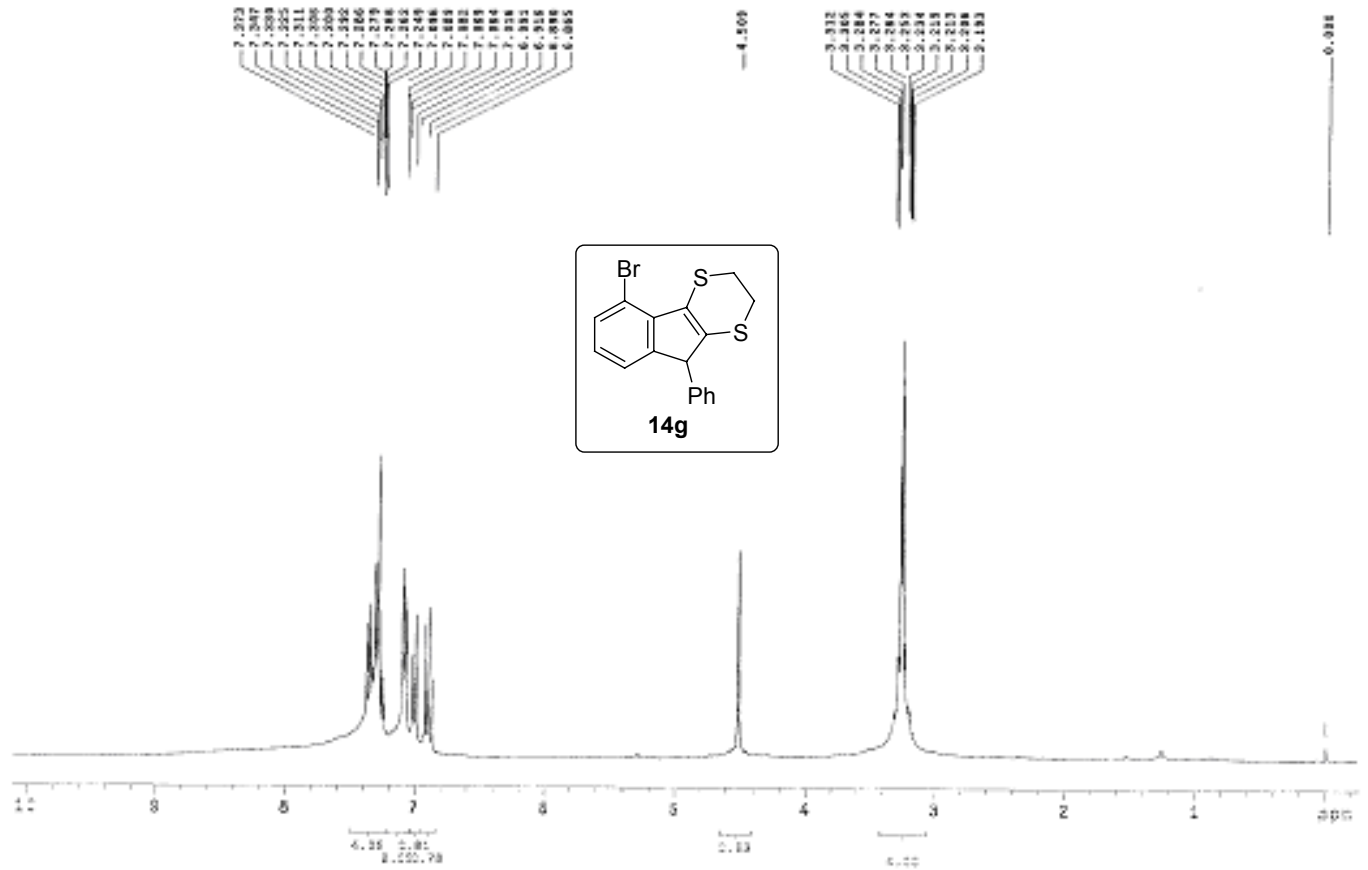

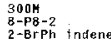

Puise Sequence: s2pu
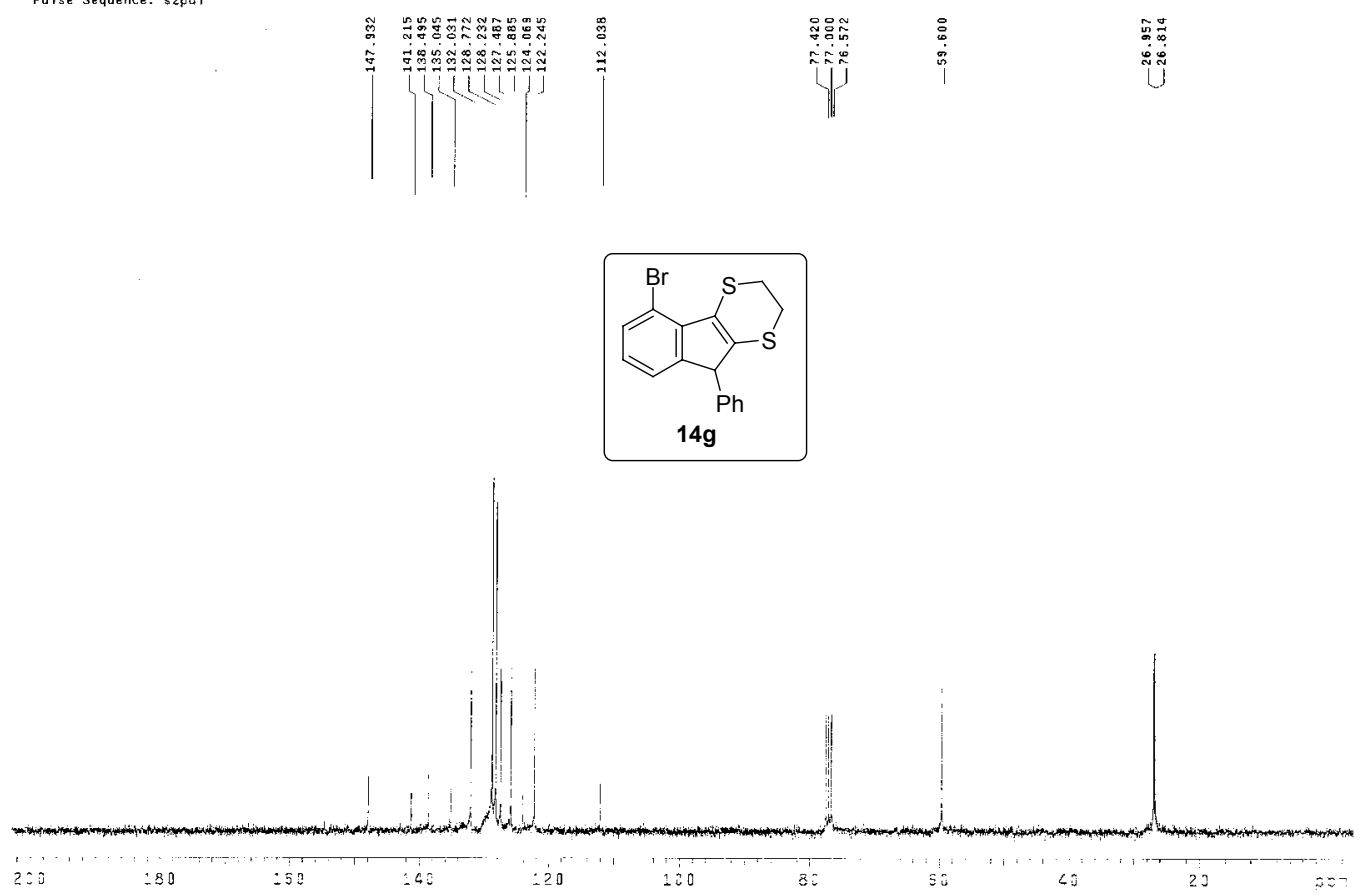
9-(4-Methylphenyl)-2,3-dihydro-1,4-dithiofluorene 14h

8004-3CHsPh-indene

Archlve directory: /export/home/vmer1/vnmisys/date

Sample directory
FIfo: PRoTok

Pulse Sequence: szpul

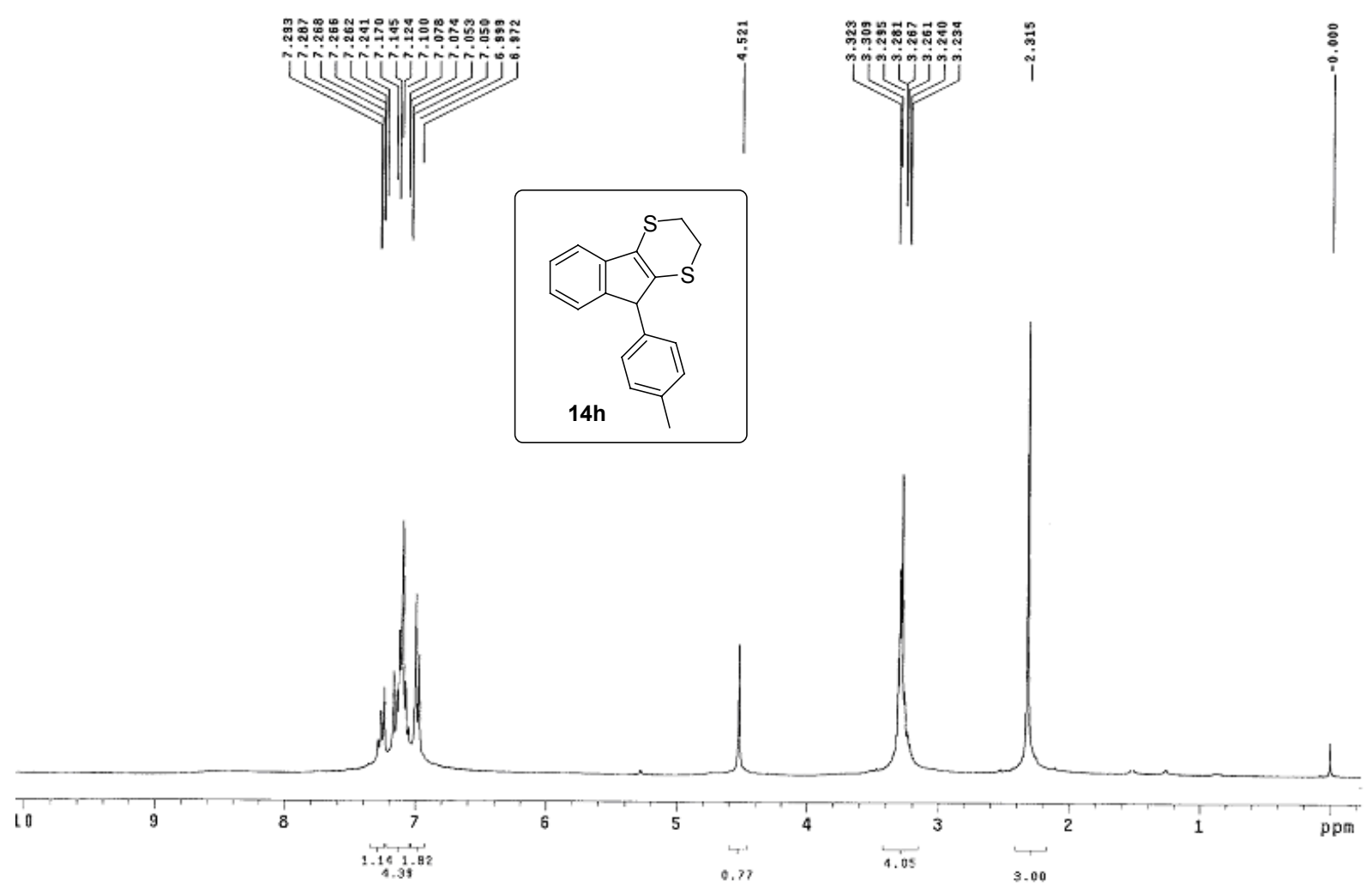

8-P5-3ch3ph-Inden

Pulse Sequence: s2pul
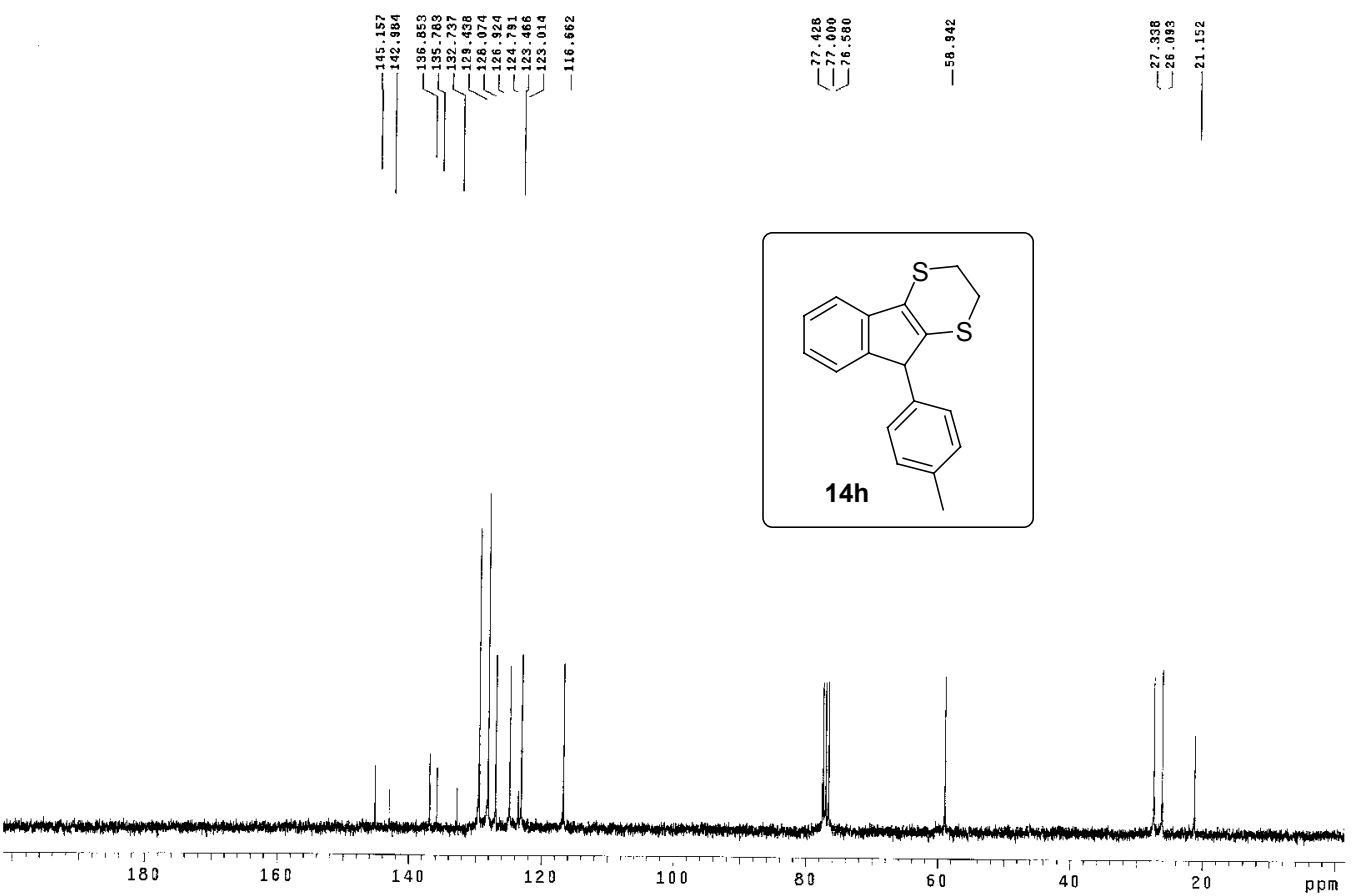
4-Phenyl-5,6-(ethylenedithiyl)cyclopentadieno[1,2-a]furan 17

soon
ruran indene
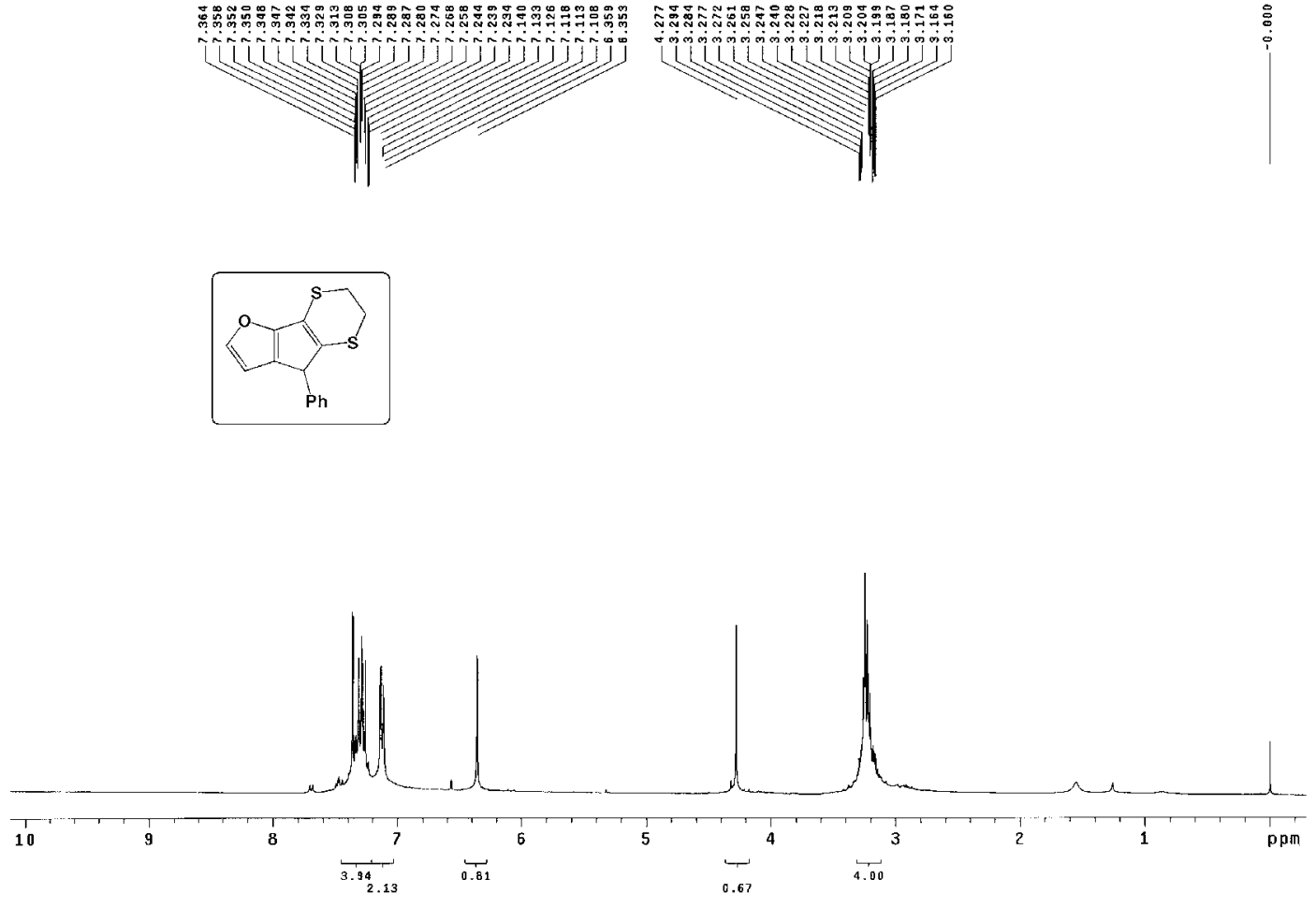

soon turan Indene
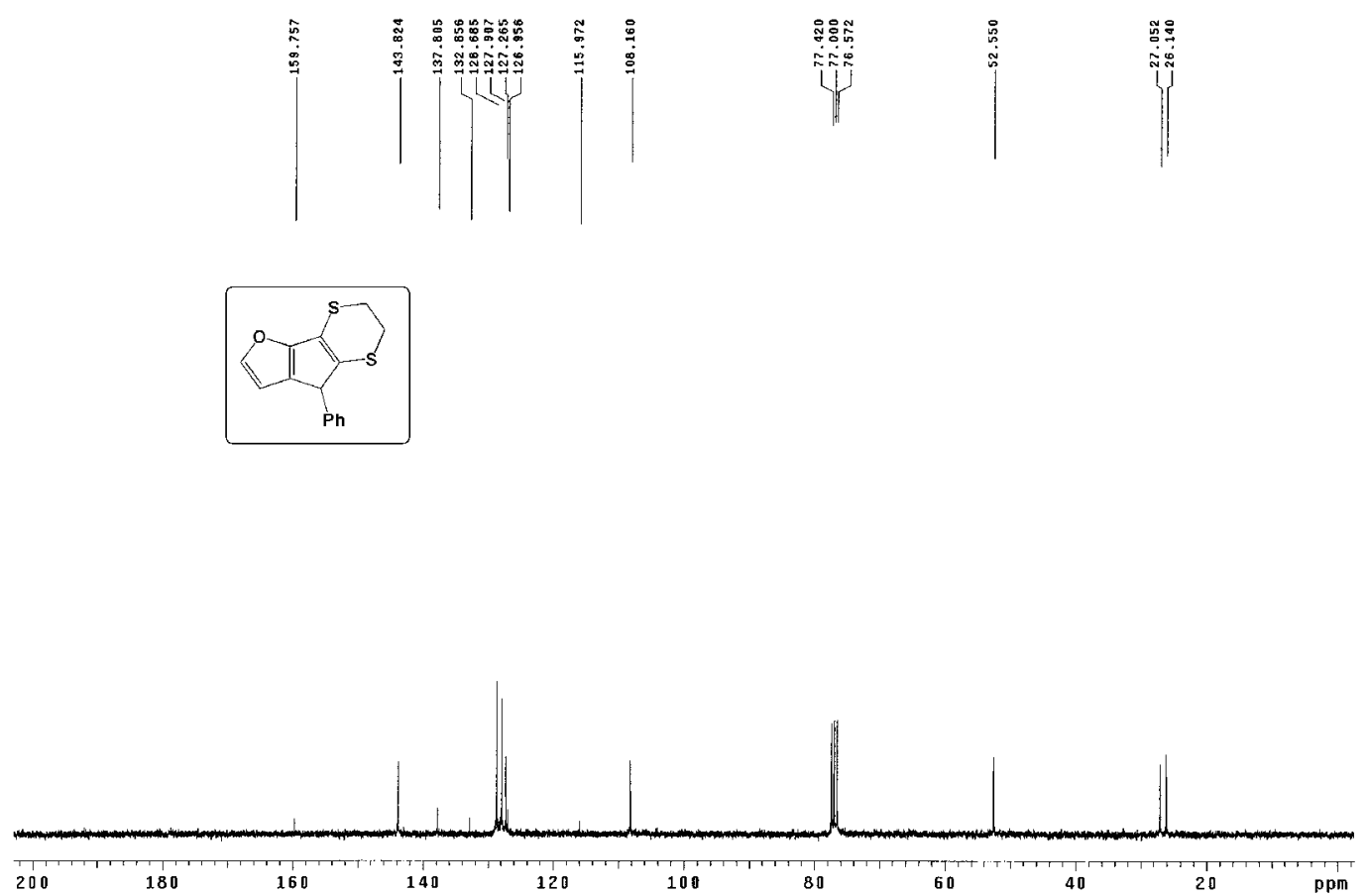


\section{Cyclopropanation products 6 (a diastereomeric mixture)}

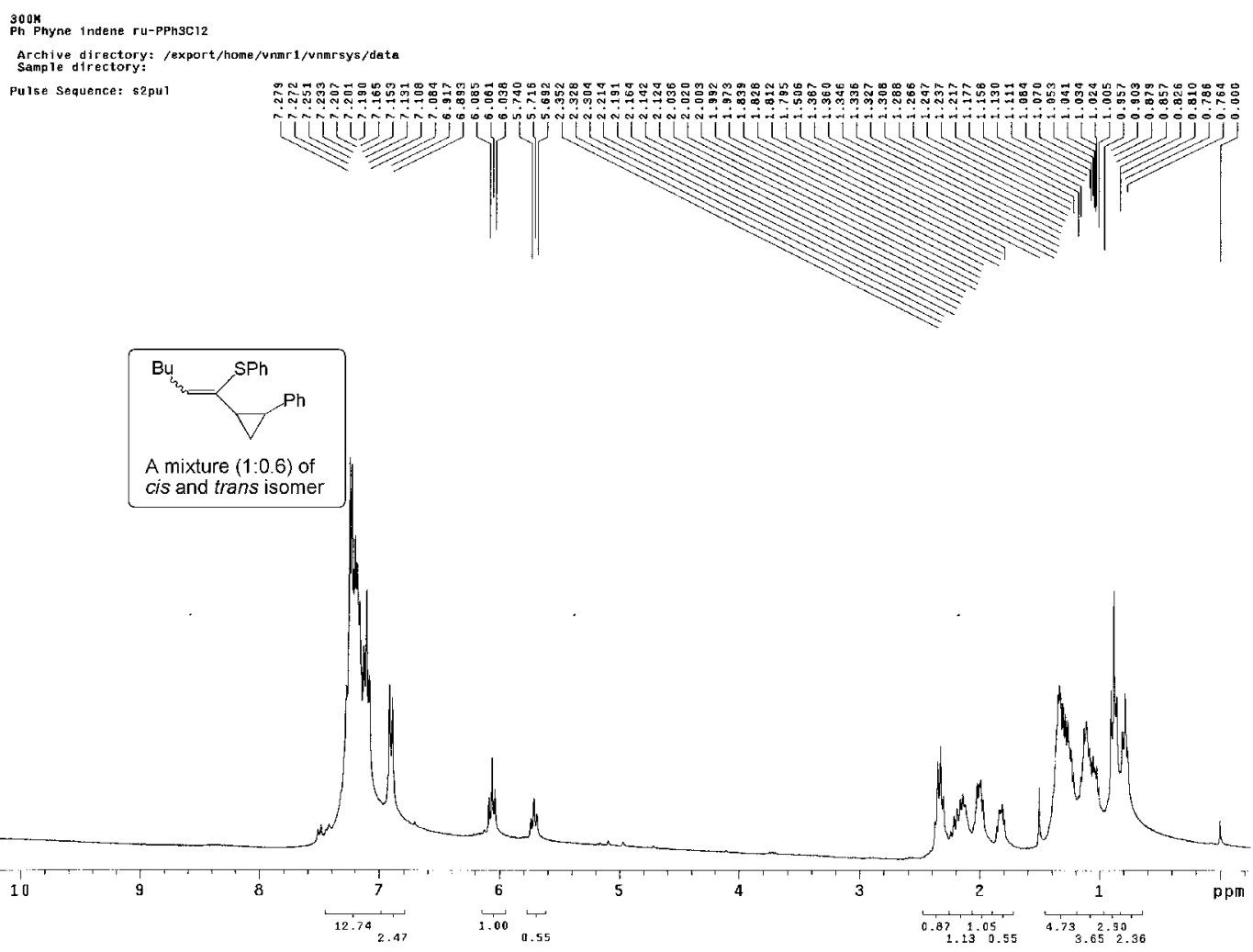

$300 \mathrm{M}$
Phene cyclopropanation Pulse sequence: szpur
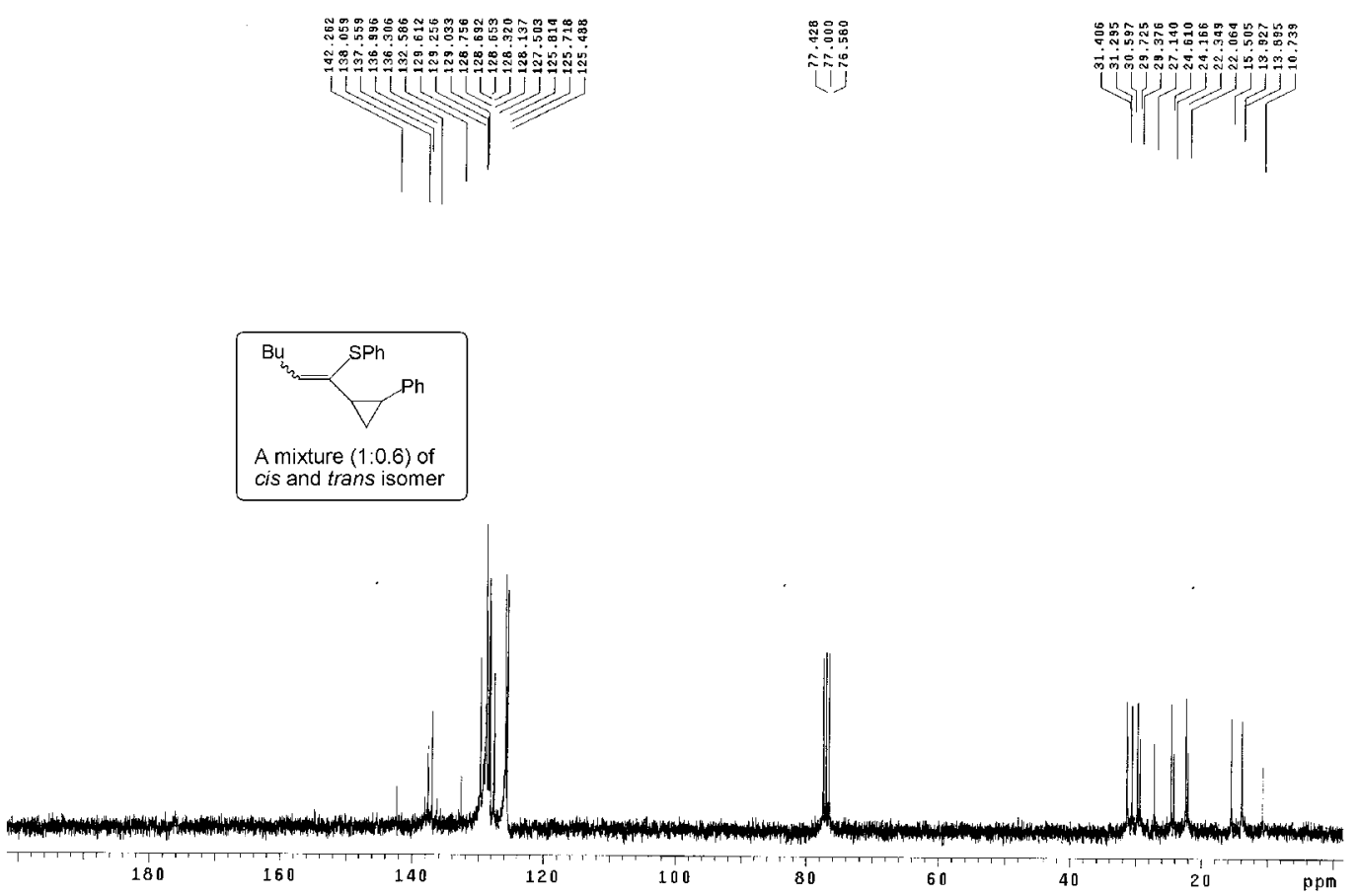
Phenyl 1-methyl-1-(2-d-phenyl)-2-propynyl sulfide 8

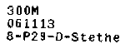

Areh1ve directory: fexport/home/vnnr 1/vnmrsys/data
Sample directory:

Pulse Sequence: $s 2 p$
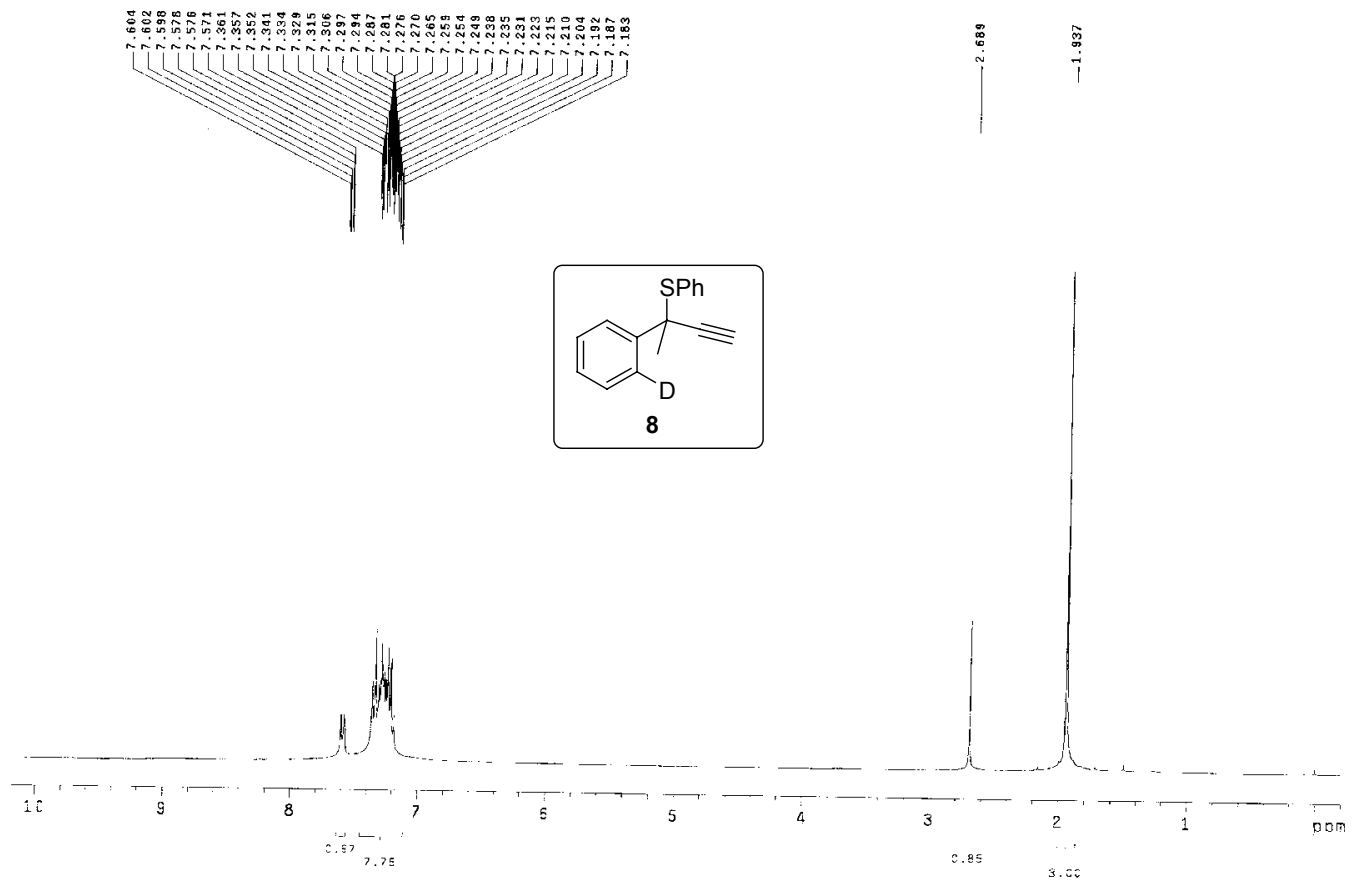

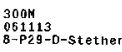

Pulse Sequence: s2pul
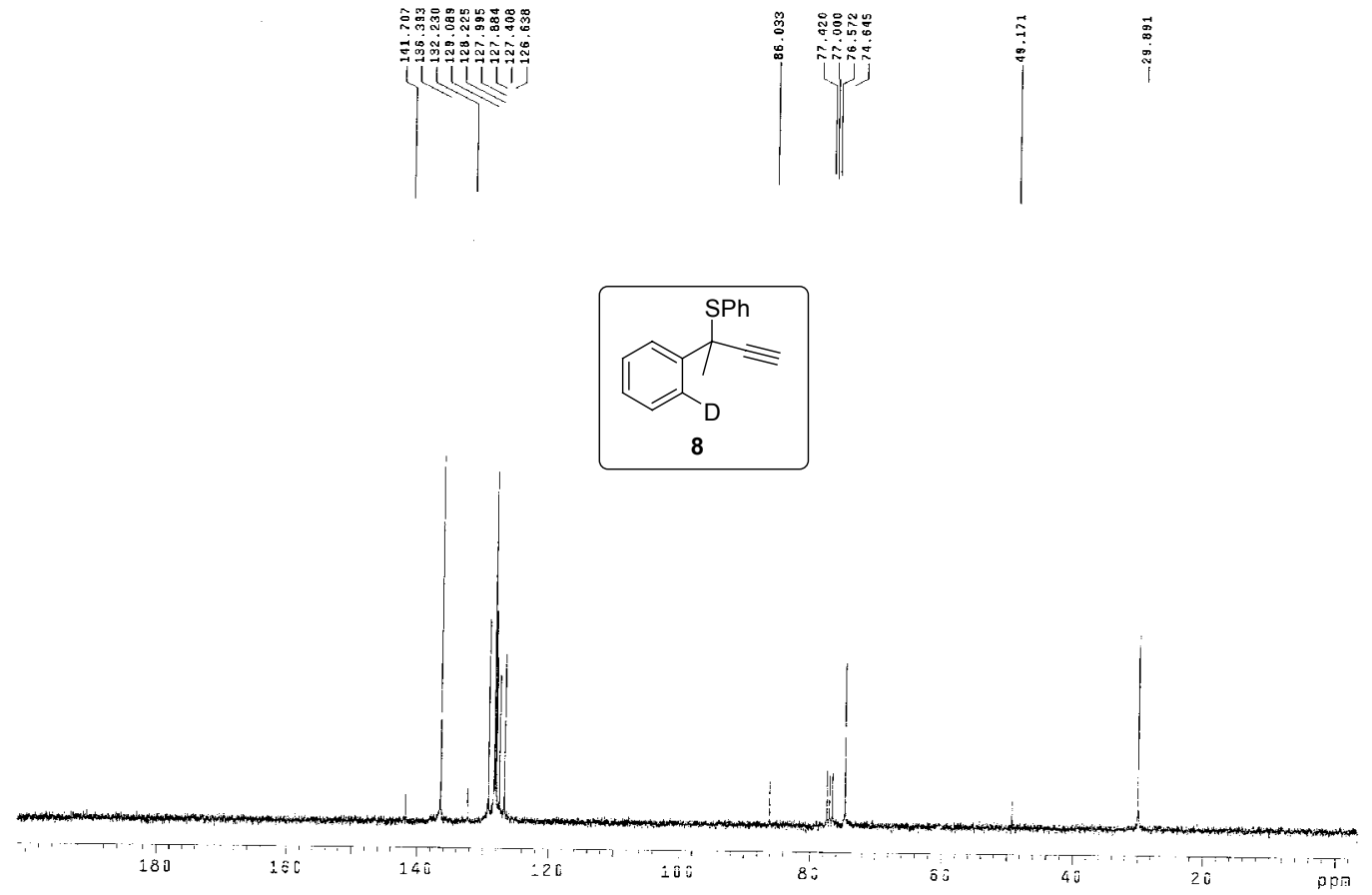


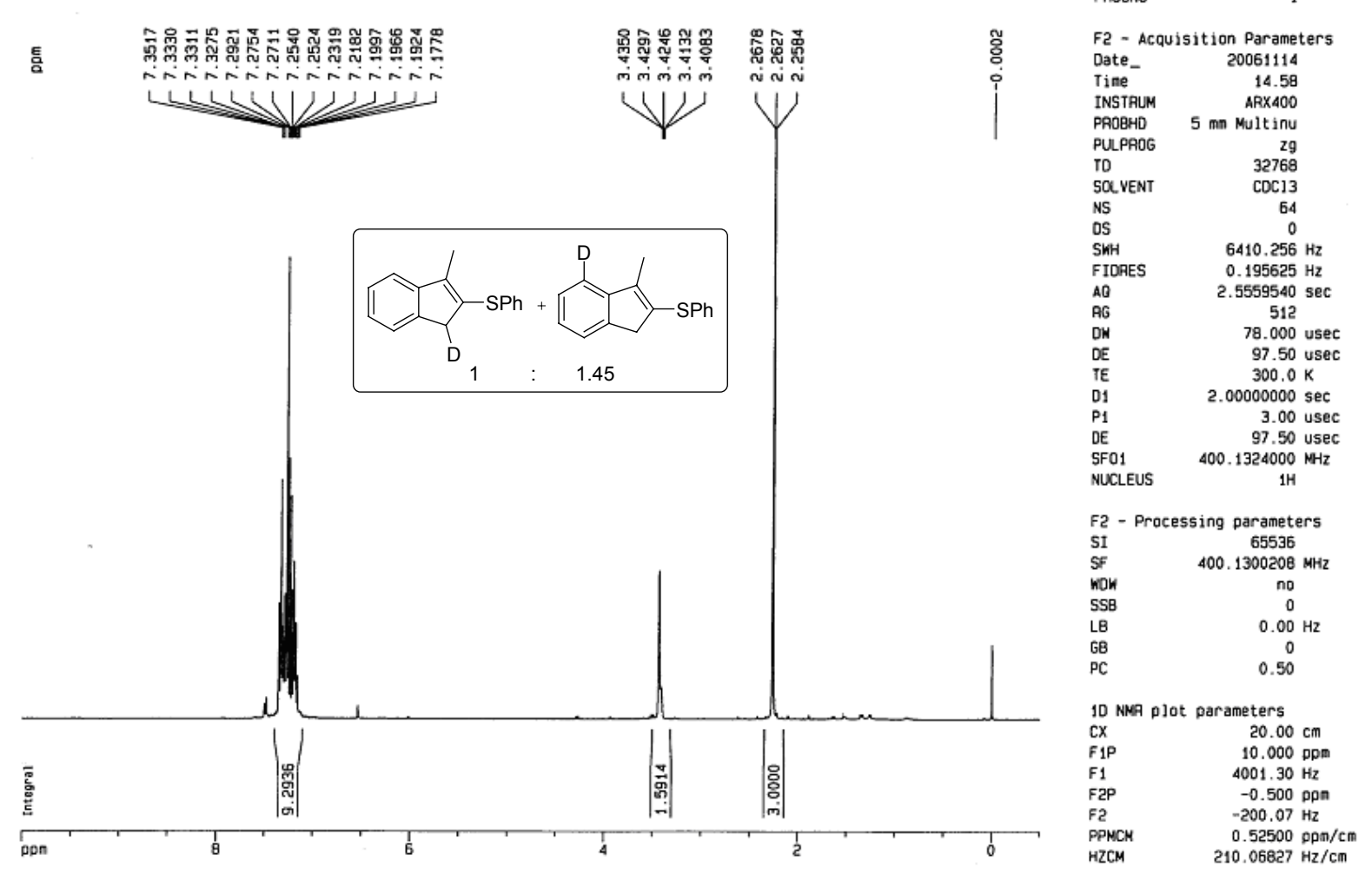

言

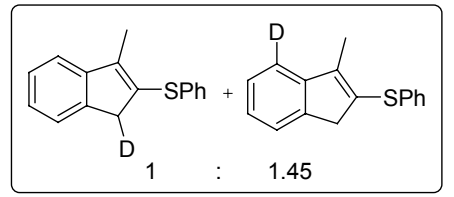

$\begin{array}{lr}\text { Current Data Parameters } \\ \text { NAME } & \text { H33012 } \\ \text { EXFNO } & 1 \\ \text { PROCNO } & 1\end{array}$

Fa - Acquisition Parameters

Date_ 20061114

Time $\quad 14.58$

INSTAUMY ARX400

PROBHO $5 \mathrm{~mm}$ Multinu

$\begin{array}{lr}\text { PULPAOG } & 29 \\ \text { TO } & 32768 \\ \text { SOLVENT } & \text { CDC13 }\end{array}$

$\begin{array}{lr}\text { SOLVENT } & \text { COC13 } \\ \text { N5 } & 64\end{array}$

OS $\quad 6410.255 \mathrm{~Hz}$

$\begin{array}{ll}\text { ShH } & 6410.256 \mathrm{~Hz} \\ \text { FIDRES } & 0.195625 \mathrm{~Hz}\end{array}$

AQ $2.5559540 \mathrm{sec}$

AG $\quad 512$

$\begin{array}{rr}\text { DW } & 78.000 \text { usec } \\ \text { DE } & 97.50 \text { usec }\end{array}$

TE $\quad 300.0 \mathrm{~K}$

$\begin{array}{ll}01 & 2.00000000 \mathrm{sec} \\ \text { P1 } & 3.00 \mathrm{usec}\end{array}$

$\begin{array}{lr}\text { DE } & 97.50 \text { usec } \\ \text { SF01 } & 400.9324000 \mathrm{uHz}\end{array}$

NUCLEUS $\quad 400.1324000 \mathrm{MHz}$

F2 - Processing parameters

$\begin{array}{lc}\text { F2 - Processing parameters } \\ \text { SI } & 65536 \\ \text { SF } & 400.130020 \mathrm{BHz} \\ \text { WDW } & \text { no } \\ \text { SSB } & 0 \\ \text { LB } & 0.00 \mathrm{~Hz} \\ \text { GB } & 0 \\ \text { PC } & 0.50\end{array}$

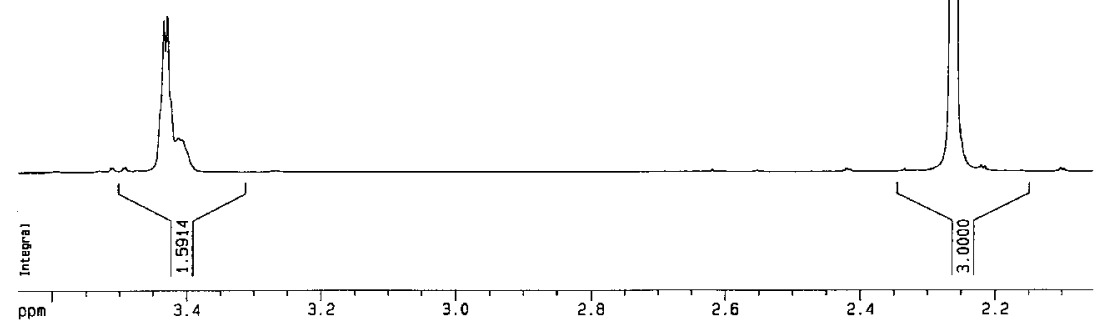

$\begin{array}{lc}\text { 10 NMR plot parameters } \\ \text { CX } & 20.00 \mathrm{~cm} \\ \text { F1P } & 3.650 \mathrm{ppm} \\ \text { F1 } & 1460.28 \mathrm{~Hz} \\ \text { F2P } & 2.054 \mathrm{gpm} \\ \text { F2 } & 822.00 \mathrm{~Hz} \\ \text { PPMCM } & 0.07976 \mathrm{ppm} / \mathrm{cm} \\ \text { HZCM } & 31.91422 \mathrm{~Hz} / \mathrm{cm}\end{array}$ 
$\underbrace{20}_{\substack{200 k \\ d-i n d e n e}}$

Puise sequence: 52pul

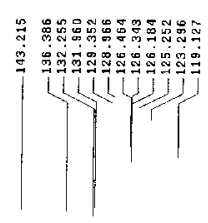

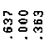

[i]
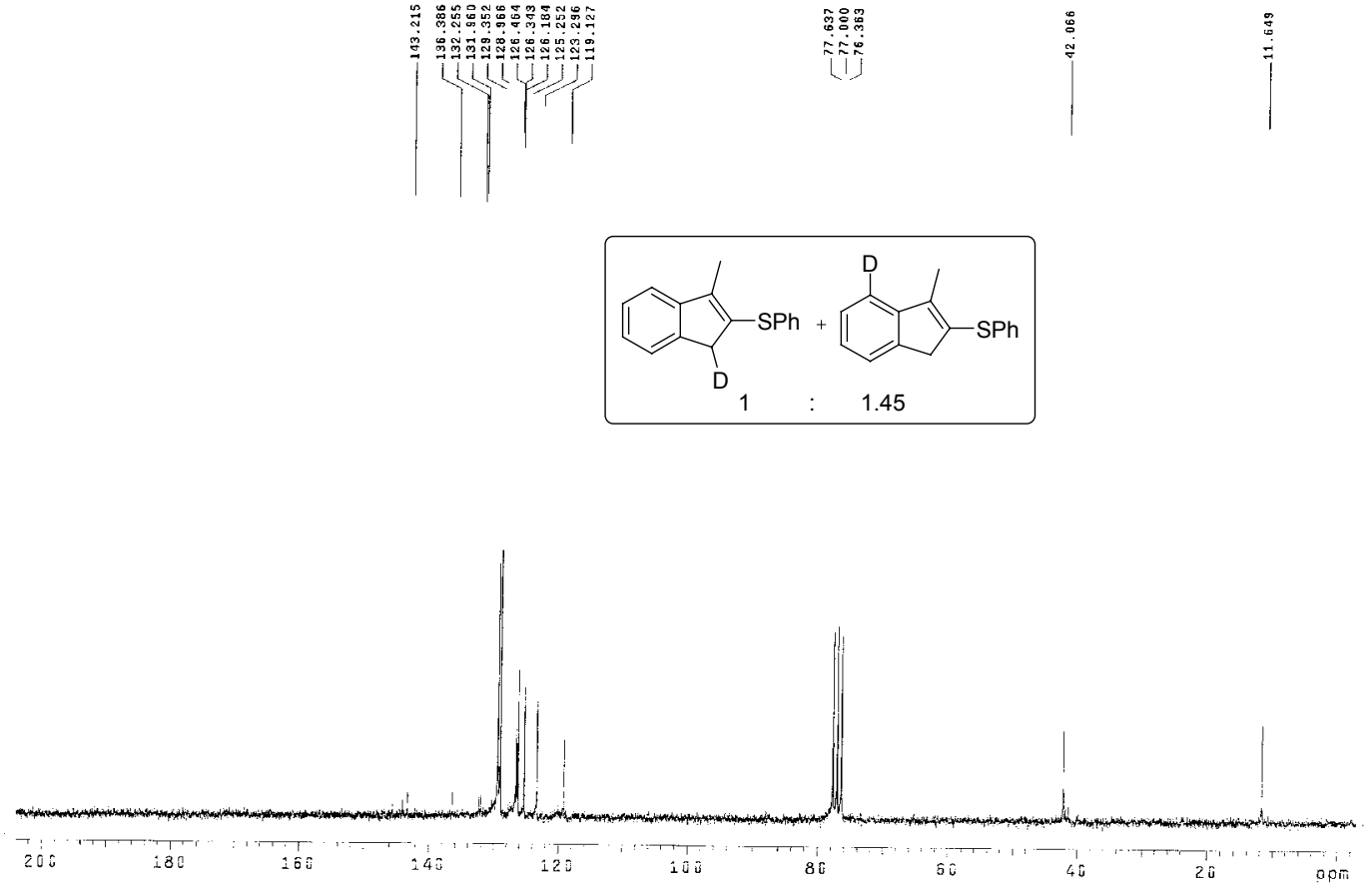